\title{
On the Hydrodynamics and Heat Convection of an Impinging External Flow Upon a Cylinder with Transpiration and Embedded in a Porous Medium
}

\author{
Rasool Alizadeh $^{1}$ - Asghar B. Rahimi ${ }^{2}$ - Nader Karimi ${ }^{3}$ • \\ Ahmad Alizadeh ${ }^{4}$
}

Received: 4 May 2017 / Accepted: 21 September 2017 / Published online: 9 October 2017

(C) The Author(s) 2017. This article is an open access publication

\begin{abstract}
This paper extends the existing studies of heat convection by an external flow impinging upon a flat porous insert to that on a circular cylinder inside a porous medium. The surface of the cylinder is subject to constant temperature and can include uniform or non-uniform transpiration. These cylindrical configurations are introduced in the analyses of stagnation-point flows in porous media for the first time. The equations governing steady transport of momentum and thermal energy in porous media are reduced to simpler nonlinear differential equations and subsequently solved numerically. This reveals the dimensionless velocity and temperature fields of the stagnation-point flow, as well as the Nusselt number and shear stress on the surface of the cylinder. The results show that transpiration on the surface of the cylinder and Reynolds number of the external flow dominate the fluid dynamics and heat transfer problems. In particular, non-uniform transpiration is shown to significantly affect the thermal and hydrodynamic responses of the system in the circumferential direction. However, the permeability and porosity of the porous medium are found to have relatively smaller influences.
\end{abstract}

Keywords Stagnation-point flow $\cdot$ Porous media $\cdot$ Semi-similar solution

\section{List of symbols}

$\begin{array}{ll}a & \text { Cylinder radius } \\ G(\eta, \varphi) & \text { Function related to } v \text {-component of velocity } \\ f(\eta, \varphi) & \text { Function related to } u \text {-component of velocity }\end{array}$

Nader Karimi

Nader.Karimi@glasgow.ac.uk

1 Department of Mechanical Engineering, Quchan Branch, Islamic Azad University, Quchan, Iran

2 Faculty of Engineering, Ferdowsi University of Mashhad, P.O. Box No. 91775-1111, Mashhad, Iran

3 School of Engineering, University of Glasgow, Glasgow G12 8QQ, UK

4 Young Researchers Club, Quchan Branch, Islamic Azad University, Quchan, Iran 


$\begin{array}{ll}h & \text { Heat transfer coefficient } \\ k & \text { Thermal conductivity } \\ \bar{k} & \text { Freestream strain rate } \\ k_{1} & \text { Permeability of the porous medium } \\ N u & \text { Nusselt number } \\ N u_{m} & \text { Averaged Nusselt number } \\ p & \text { Fluid pressure } \\ P_{0} & \text { Non-dimensional fluid pressure at stagnation point } \\ P & \text { Non-dimensional fluid pressure } \\ P r & \text { Prandtl number } \\ q_{\mathrm{w}} & \text { Heat flow at wall } \\ r & \text { Radial coordinate } \\ R e & \text { Freestream Reynolds number } \operatorname{Re}=\frac{\bar{k} a^{2}}{2 v} \\ S(\varphi) & \text { Transpiration rate function } S(\varphi)=\frac{U_{0}(\varphi)}{\bar{k} a} \\ T & \text { Temperature } \\ T_{\infty} & \text { Freestream temperature } \\ T_{\mathrm{W}} & \text { Wall temperature } \\ u, v, w & \text { Velocity components along } r-, \varphi-, z \text {-axis } \\ U_{0}(\varphi) & \text { Transpiration } \\ z & \text { Axial coordinate }\end{array}$

\section{Greek symbols}

$\begin{array}{ll}\alpha & \text { Effective thermal diffusivity of the porous medium } \\ \eta & \text { Similarity variable } \\ \varepsilon & \text { Porosity } \\ \theta & \text { Non-dimensional temperature } \\ \lambda & \text { Reciprocal of Darcy number } \lambda=\frac{a^{2}}{4 k_{1}} \\ \mu & \text { Dynamic viscosity } \\ v & \text { Kinematic viscosity } \\ \rho & \text { Fluid density } \\ \sigma & \text { Shear stress } \\ \sigma_{m} & \text { Averaged shear stress } \\ \varphi & \text { Angular coordinate }\end{array}$

\section{Introduction}

Stagnation-point flows over solid surfaces have attracted attention of engineers and scientists for more than a century (Hiemenz 1911). This is due to the appearance of such flow configurations in a large number of natural and industrial applications. Most notably, significant enhancements of heat transfer by impinging jets are well demonstrated and these flows are widely used in process and manufacturing industries (Rohsenow et al. (1998)). Classical investigation of an impinging flow was first conducted by Hiemenz (1911) through finding an exact, two-dimensional solution of the Navier-Stokes equations near the stagnation point on a flat plate. This solution was later completed by Homann (1936) and also Howarth (1951) and Davey (1961). By employing numerical methods, these studies have been further advanced to unsteady cases on flat plates with uniform or non-uniform transpiration (Rahimi 
and Abbassi 2009; Abbassi et al. 2011; Abbassi and Rahimi 2012). The theoretical treatments of stagnation-point flows were extended to cylindrical configurations in the seventies (Wang 1973, 1974). More recent studies of flow impingement on cylinders included the effects of axial motion (Saleh and Rahimi 2004), transpiration (Rahimi and Saleh 2007) and unsteady angular motion of the curved surface (Rahimi and Saleh 2008).

The general problem of flow impingement on a porous medium has received a considerable attention from the research community. The hydrodynamics of stagnation-point, isothermal flow on a flat porous insert was analysed theoretically by Wu et al. (2005). Through assuming a Darcy-Brinkman flow, Wu et al. developed an asymptotic solution for the velocity field inside a horizontal porous medium interacting with a downward impinging jet (Wu et al. 2005). They identified a transition in the flow behaviour on the basis of a parameter defined as the ratio of the kinematic viscosity to the product of the permeability of the medium and a characteristic velocity gradient (Wu et al. 2005). In a numerical study Jeng and Tzeng (2005) simulated the heat transfer process during impingement of a slot jet on the surface of a metallic foam heat sink. These authors found that the location of the maximum Nusselt number is dependent upon the jet Reynolds number (Jeng and Tzeng 2005). They also showed that this location moves from somewhere close to the stagnation point at low Reynolds number to further inside the porous medium at higher values of Reynolds number (Jeng and Tzeng 2005). Later, the same group of authors conducted an experimental investigation on the problem of jet impingement on a porous block (Jeng and Tzeng 2007). Temperature and pressure measurements revealed that by increasing the jet Reynolds number Nusselt number increases, while the flow pressure drop is also intensified (Jeng and Tzeng 2007). Wong and Saeid (2009a, b) considered a jet flow blowing on the surface of a horizontal porous medium heated from below and investigated the heat transfer by mixed convection. By assuming local thermal equilibrium (Wong and Saeid 2009a) and local thermal non-equilibrium (Wong and Saeid 2009b) they conducted numerical parametric studies to optimise the Nusselt number.

A similarity solution was developed by Harris et al. (2009) for mixed convection boundary layer formed near the stagnation point on a vertical porous insert. This study further included a stability analysis of the developed solution (Harris et al. 2009). The problem of mixed convection in jet impingement on a flat porous medium was also investigated numerically by Sivasamy et al. (2010). In this work, a constant heat flux was applied to the bottom of the porous insert in an axisymmetric configuration (Sivasamy et al. 2010). The analysis showed that increasing the jet width and the Reynolds number results in magnifying the average Nusselt number (Sivasamy et al. 2010). It also revealed that decreasing the distance between the jet and the heated section leads to an increase in the Nusselt number (Sivasamy et al. 2010). Analytical solutions were developed by Kokubun and Fachini (2011) for the stagnation-point flow in an infinitely long, horizontal porous insert. This configuration was subject to constant wall heat flux and constant wall temperature boundary conditions at the bottom plate and could be considered as the heat transferring version of the problem solved by $\mathrm{Wu}$ et al. (2005). The work of Kokubun and Fachini (2011) showed that a dimensionless parameter, containing information on the transport characteristics of the fluid and solid phase, governs the behaviour of heat transferring flow. This finding was analogous to that of Wu et al. (2005) in the corresponding isothermal flow. Feng et al. (2014) tackled the problem of tube flow impingement on a heated porous block experimentally and numerically. They investigated metal foam and finned metal foams and showed that by increasing the height of metal foam the Nusselt number decreases, while this was not the case for the metal finned foam (Feng et al. 2014). They further reported that under the same flow conditions the heat transfer rate in the metal finned foam was up to 2.8 times greater than that in the metal foam (Feng et al. 2014). Most recently, Buonomo et al. (2016) examined the interactions between a downward 
vertical, laminar jet and a confined, horizontal porous layer in an axisymmetric configuration. Their numerical study showed that Peclet number is the main parameter in determining the opposing or supporting arrangements of natural and forced convection (Buonomo et al. 2016).

The preceding discussion reveals that the problem of interactions between an impinging flow and a flat porous medium is relatively well studied. A common feature amongst most of the existing works in this area is the existence of an impermeable surface under a horizontal or vertical porous plate. Consideration of transpiration in the form of fluid suction or blowing is limited to a few theoretical investigations. Ishak et al. (2008) developed a similarity solution for an external flow impinging on a vertical porous wall, which allows mass exchanges. A similar flow configuration was analysed theoretically by Hamad and Pop (2011). These authors considered nanofluid convection and set the system to have internal heat generation or consumption and showed that nanofluid flow patterns can be different to those observed in ordinary fluids (Hamad and Pop 2011). This configuration was subsequently investigated by Hamad and Ferdows (2012), who applied Lie group analysis and conducted a comprehensive parametric study. The effects of thermal radiation on the problem of stagnation-point flow over a porous shrinking sheet with suction/blowing were examined theoretically by Bhattacharyya and Layek (2011). Later, Rohni et al. (2012) investigated the unsteady mixed convection caused by a stagnation-point flow over a flat plate embedded in porous media. They let the porous medium feature fluid suction and temperature slip and developed similarity solutions for the flow and temperature fields. In particular, these authors showed that multiple solutions existed for specific ranges of parameters.

It follows from the survey of literature that, so far, all studies of stagnation-point flows over porous media have been exclusively concerned with flat porous inserts. Further, they have been largely focused on the cases with no transpiration. Curved porous systems with or without transpiration have been rarely investigated. Given the practical significance of curved porous media, particularly around circular tubes, the lack of studies in this area is surprising. The current work, therefore, aims to fill this gap through considering a stagnation-point flow on the surface of a cylinder embedded in porous media, which may also include transpiration. Constant temperature boundary conditions are applied on the surface of the cylinder, and the steady flow and temperature fields along with Nusselt number and imposed shear stress are calculated. This investigation will be an extension of the analyses of Wu et al. (2005) and Kokubun and Fachini (2011) to cylindrical configurations with the inclusion of non-uniform transpiration.

\section{Theoretical Method}

\subsection{Problem Configuration and Governing Equations}

Figure 1 shows the schematic view of the problem under investigation. This includes a cylinder with radius $a$ centred at $r=0$, which is embedded in a porous medium. The cylinder can feature a uniform or non-uniform transpiration with prescribed circumferential distributions. Further, the external surface of the cylinder is subject to constant temperature boundary condition. An external axisymmetric radial stagnation-point flow of strain rate of $\bar{k}$ impinges upon the cylinder. Due to the non-uniformity of transpiration the overall flow configuration can be un-axisymmetric. The following assumptions are made throughout the current investigation.

- The fluid is Newtonian, and the flow is steady, incompressible and laminar. 


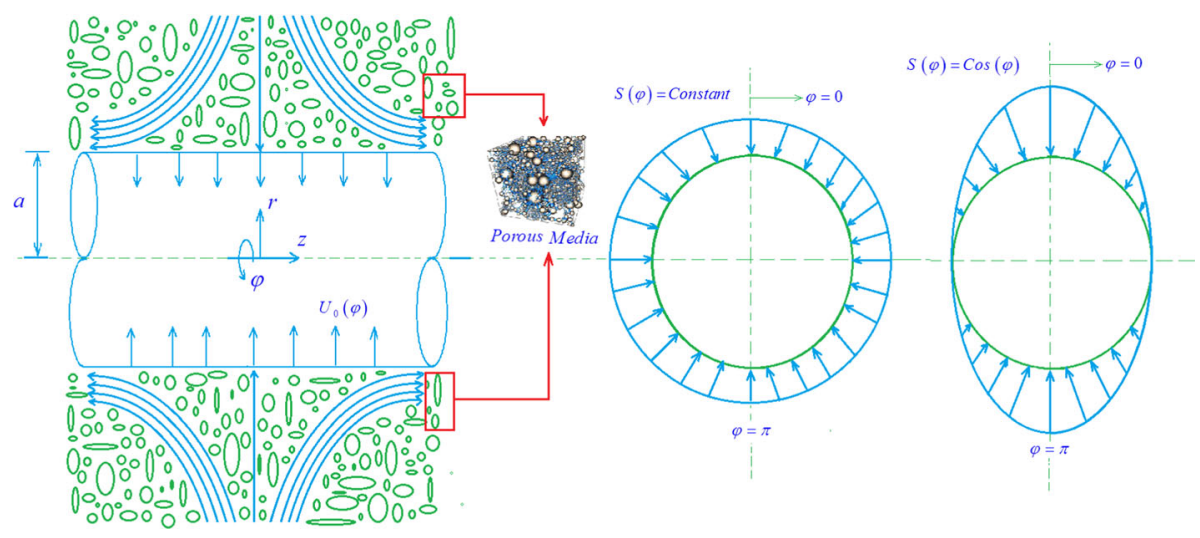

Fig. 1 Schematic view of the problem under investigation

- The cylinder is assumed to be infinitely long.

- The porous medium is homogenous, isotropic and under local thermal equilibrium, and hence local thermal non-equilibrium effects (Nazari et al. 2013) are ignored.

- The radiation and natural convection modes of heat transfer are ignored.

- The viscous dissipation of the flow kinetic energy is ignored.

- Physical properties such as porosity, specific heat, density and thermal conductivity are assumed to be constant, and hence the thermal dispersion effects are ignored.

- A moderate range of pore-scale Reynolds number is considered in the porous medium, and therefore nonlinear effects in momentum transfer are negligibly small.

A two-dimensional Darcy-Brinkman model of transport of momentum along with the oneequation model of transport of thermal energy in cylindrical coordinate is employed in this study (Nield and Bejan 2006; Joseph et al. 1982; Wu et al. 2005). The following summarises the governing equations and the corresponding boundary conditions in the cylindrical coordinate system shown in Fig. 1.

The continuity of mass is

$$
\frac{\partial(r u)}{\partial r}+\frac{\partial v}{\partial \varphi}+r \frac{\partial w}{\partial z}=0
$$

The transport of momentum in the radial direction is written as

$$
\frac{\rho}{\varepsilon^{2}}\left(u \frac{\partial u}{\partial r}+\frac{v}{r} \frac{\partial u}{\partial \varphi}-\frac{v^{2}}{r}+w \frac{\partial u}{\partial z}\right)=-\frac{\partial p}{\partial r}+\frac{\mu}{\varepsilon}\left[\frac{\partial^{2} u}{\partial r^{2}}+\frac{1}{r} \frac{\partial u}{\partial r}-\frac{u}{r^{2}}+\frac{1}{r^{2}} \frac{\partial^{2} u}{\partial \varphi^{2}}+\frac{\partial^{2} u}{\partial z^{2}}\right]-\frac{\mu}{k_{1}} u,
$$

that in the circumferential direction is given by

$$
\begin{aligned}
& \frac{\rho}{\varepsilon^{2}}\left(u \frac{\partial v}{\partial r}+\frac{v}{r} \frac{\partial v}{\partial \varphi}+\frac{u v}{r}+w \frac{\partial v}{\partial z}\right) \\
& =-\frac{\partial p}{r \partial \varphi}+\frac{\mu}{\varepsilon}\left[\frac{\partial^{2} v}{\partial r^{2}}+\frac{1}{r} \frac{\partial v}{\partial r}-\frac{v}{r^{2}}+\frac{1}{r^{2}} \frac{\partial^{2} v}{\partial \varphi^{2}}+\frac{2}{r^{2}} \frac{\partial u}{\partial \varphi}+\frac{\partial^{2} v}{\partial z^{2}}\right]-\frac{\mu}{k_{1}} v
\end{aligned}
$$


and the transport of momentum in the axial direction takes the form of

$$
\frac{\rho}{\varepsilon^{2}}\left(u \frac{\partial w}{\partial r}+\frac{v}{r} \frac{\partial w}{\partial \varphi}+w \frac{\partial w}{\partial z}\right)=-\frac{\partial p}{\partial z}+\frac{\mu}{\varepsilon}\left[\frac{\partial^{2} w}{\partial r^{2}}+\frac{1}{r} \frac{\partial w}{\partial r}+\frac{1}{r^{2}} \frac{\partial^{2} w}{\partial \varphi^{2}}+\frac{\partial^{2} w}{\partial z^{2}}\right]-\frac{\mu}{k_{1}} w .
$$

The transport of thermal energy is expressed by

$$
u \frac{\partial T}{\partial r}+\frac{v}{r} \frac{\partial T}{\partial \varphi}+w \frac{\partial T}{\partial z}=\alpha\left[\frac{\partial^{2} T}{\partial r^{2}}+\frac{1}{r} \frac{\partial T}{\partial r}+\frac{1}{r^{2}} \frac{\partial^{2} T}{\partial \varphi^{2}}+\frac{\partial^{2} T}{\partial z^{2}}\right] .
$$

In Eqs. (1-5) $p, \rho, v, T$ and $k_{1}$ are the fluid pressure, density, kinematic viscosity, temperature and permeability of the porous medium, respectively. These properties are evaluated inside the boundary layer and in the vicinity of the flow impingement point. The boundary conditions for the velocity field are as follows:

$$
\begin{aligned}
r=a: w & =0, \quad v=0, \quad u=-U_{0}(\varphi) \\
r \rightarrow \infty: w & =2 \bar{k} z, \quad \lim _{r \rightarrow \infty} r v=0, \quad u=-\bar{k}\left(r-\frac{a^{2}}{r}\right) .
\end{aligned}
$$

Further, the two boundary conditions with respect to $\varphi$ (angular coordinate) are

$$
\begin{gathered}
u(r, 0)=u(r, 2 \pi), v(r, 0)=v(r, 2 \pi), \\
\frac{\partial u(r, 0)}{\partial \varphi}=\frac{\partial u(r, 2 \pi)}{\partial \varphi}, \frac{\partial v(r, 0)}{\partial \varphi}=\frac{\partial v(r, 2 \pi)}{\partial \varphi} .
\end{gathered}
$$

Equation (6) represents no-slip conditions on the external surface of the cylinder. Also relations (7) show that the viscous flow solution approaches, in a manner analogous to the Hiemenz flow, the potential flow solution as $r \rightarrow \infty$ Alizadeh et al. (2016a, b). This can be confirmed by starting from the continuity equation in the following. $-\frac{1}{r} \frac{\partial}{\partial r}(r u)-\frac{\partial v}{\partial \varphi}=\frac{\partial w}{\partial z}=$ Constant $=2 \bar{k} z$, and integrating in $r$-and $z$-directions with boundary conditions, $w=0$ when $z=0$ and $u=-U_{0}(\varphi)$ when $r=a$.

The boundary conditions for the transport of energy take the form of

$$
\begin{aligned}
& r=a: \quad T=T_{\mathrm{W}}=\text { Constant, } \\
& r \rightarrow \infty: \quad T \rightarrow T_{\infty},
\end{aligned}
$$

and the two boundary conditions with respect to angular coordinate, $\varphi$, are

$$
\begin{aligned}
T(r, 0) & =T(r, 2 \pi), \\
\frac{\partial T(r, 0)}{\partial \varphi} & =\frac{\partial T(r, 2 \pi)}{\partial \varphi},
\end{aligned}
$$

where $T_{\mathrm{W}}$ is the cylinder surface temperature and $T_{\infty}$ is the freestream temperature.

\subsection{Self-similar Solutions}

A reduction of the governing Eqs. (1-4) is obtained through applying the following similarity transformations.

$$
u=-\frac{\bar{k} a}{\sqrt{\eta}} f(\eta, \varphi), v=\frac{\bar{k} a}{\sqrt{\eta}} G(\eta, \varphi), w=\left[2 \bar{k} f^{\prime}(\eta, \varphi)-\frac{\bar{k}}{\eta} \frac{\partial G}{\partial \varphi}\right] z, p=\rho \bar{k}^{2} a^{2} P,
$$


where $\eta=\left(\frac{r}{a}\right)^{2}$ is the dimensionless radial variable. Transformations (11) satisfy Eq. (1) automatically, and their substitution into Eqs. (2), (3) and (4) yields a coupled system of differential equations in terms of $f(\eta, \varphi)$, and an expression for the pressure:

$$
\begin{aligned}
\varepsilon . & \left(\eta f^{\prime \prime \prime}+f^{\prime \prime}-\frac{1}{8 \eta^{2}} \frac{\partial^{3} G}{\partial \varphi^{3}}-\frac{1}{2} \frac{\partial G^{\prime \prime}}{\partial \varphi}+\frac{1}{2 \eta} \frac{\partial G^{\prime}}{\partial \varphi}-\frac{1}{2 \eta^{2}} \frac{\partial G}{\partial \varphi}+\frac{1}{4 \eta} \frac{\partial^{2} f^{\prime}}{\partial \varphi^{2}}\right) \\
& +\operatorname{Re}\left[1+f f^{\prime \prime}-\left(f^{\prime}\right)^{2}-\frac{f}{2 \eta} \frac{\partial G^{\prime}}{\partial \varphi}+\frac{f}{2 \eta^{2}} \frac{\partial G}{\partial \varphi}-\frac{G}{2 \eta} \frac{\partial f^{\prime}}{\partial \varphi}+\frac{G}{4 \eta^{2}} \frac{\partial^{2} G}{\partial \varphi^{2}}+\frac{f^{\prime}}{\eta} \frac{\partial G}{\partial \varphi}\right. \\
& \left.-\frac{1}{4 \eta^{2}}\left(\frac{\partial G}{\partial \varphi}\right)^{2}\right]+\varepsilon^{2} \cdot \lambda\left[1-f^{\prime}\right]=0 \\
P & -P_{0}=-\frac{1}{2 \varepsilon^{2}}\left(\frac{f^{2}}{\eta}\right)-\frac{1}{\varepsilon}\left(\frac{f^{\prime}}{R e}\right)+\frac{1}{\varepsilon^{2}} \frac{1}{2 \bar{k}^{2}} \int_{1}^{\eta} \frac{1}{\eta^{2}}\left[G^{2}+G \frac{\partial f}{\partial \varphi}\right] \mathrm{d} \eta \\
& -\frac{1}{\varepsilon} \frac{1}{2 R e} \int_{1}^{\eta}\left[\frac{1}{2 \eta^{2}} \frac{\partial^{2} f}{\partial \varphi^{2}}+\frac{1}{\eta^{2}} \frac{\partial G}{\partial \varphi}\right] \mathrm{d} \eta-2\left[\frac{1}{\varepsilon^{2}}+\frac{\lambda}{R e}\right]\left(\frac{z}{a}\right)^{2},
\end{aligned}
$$

where $R e=\frac{\bar{k} a^{2}}{2 v}$ is the freestream Reynolds number, $\lambda=\frac{a^{2}}{4 k_{1}}$ is the reciprocal of Darcy number, and prime indicates differentiation with respect to $\eta$. Using conditions (6), (7) and (8) the boundary conditions for Eqs. (12) and (13) can be derived as follows:

$$
\begin{aligned}
& \eta=1: \quad f^{\prime}(1, \varphi)=0, f(1, \varphi)=S(\varphi) \\
& \eta \rightarrow \infty: \quad f^{\prime}(\infty, \varphi)=1 \\
& f(\eta, 0)=f(\eta, 2 \pi), \quad \frac{\partial f(\eta, 0)}{\partial \varphi}=\frac{\partial f(\eta, 2 \pi)}{\partial \varphi}
\end{aligned}
$$

in which $S(\varphi)=\frac{U_{0}(\varphi)}{\bar{k} a}$ is the transpiration rate function. Note that Eqs. (12) and (13) are the complete form of Eqs. (9) and (11) in Saleh and Rahimi (2004). Insertion of transformations (11) into Eqs. (3) and (4) yields a differential equation in terms of $G(\eta, \varphi)$ as well as an expression for the pressure. This is

$$
\varepsilon \cdot\left(\eta G^{\prime \prime}+\frac{1}{4 \eta} \frac{\partial^{2} G}{\partial \varphi^{2}}-\frac{1}{2 \eta} \frac{\partial f}{\partial \varphi}\right)+\operatorname{Re}\left[f \cdot G^{\prime}-\frac{G}{2 \eta} \frac{\partial G}{\partial \varphi}\right]-\varepsilon^{2} \cdot \lambda \cdot G=0,
$$

Considering conditions (6)-(9), the boundary and initial conditions for Eq. (17) can be written as

$$
\begin{gathered}
\eta=1: \quad G(1, \varphi)=0, \frac{\partial G(1, \varphi)}{\partial \varphi}=0 \\
\eta \rightarrow \infty: \quad G(\infty, \varphi)=0 \\
G(\eta, 0)=G(\eta, 2 \pi), \frac{\partial G(\eta, 0)}{\partial \varphi}=\frac{\partial G(\eta, 2 \pi)}{\partial \varphi} .
\end{gathered}
$$

To transform the energy Eq. (5) into a non-dimensional form, the following transformation is introduced (Alizadeh et al. $(2016 \mathrm{~b}, \mathrm{c})$ ).

$$
\theta(\eta, \varphi)=\frac{T(\eta, \varphi)-T_{\infty}}{T_{\mathrm{W}}-T_{\infty}}
$$

Substitution of Eqs. (11) and (20) into Eq. (5) and neglecting the small dissipation terms result in

$$
\eta \theta^{\prime \prime}+\theta^{\prime}+\frac{1}{4 \eta} \frac{\partial^{2} \theta}{\partial \varphi^{2}}+R e \cdot \operatorname{Pr}\left(f \theta^{\prime}-\frac{G}{2 \eta} \frac{\partial \theta}{\partial \varphi}\right)=0
$$


with the associated boundary conditions written as

$$
\begin{gathered}
\eta=1: \quad \theta(1, \varphi)=1 \\
\eta \rightarrow \infty: \quad \theta(\infty, \varphi)=0 \\
\theta(r, 0)=\theta(r, 2 \pi), \frac{\partial \theta(r, 0)}{\partial \varphi}=\frac{\partial \theta(r, 2 \pi)}{\partial \varphi} .
\end{gathered}
$$

It should be noted that Eq. (17) is the complete form of Eqs. (14) in Saleh and Rahimi (2004). Equations (12), (17) and (21), along with the boundary conditions (14-16), (1819), (22) and (23), were solved numerically by utilising an implicit, iterative tri-diagonal, finite difference method similar to that discussed by Ganesan and Palani (2004) and Thomas (2013). Although not shown here, the obtained results revealed that the dimensionless velocity component in the circumferential direction is negligible. Hence, in the rest of this paper we assume that $G(\eta, \varphi)=0$.

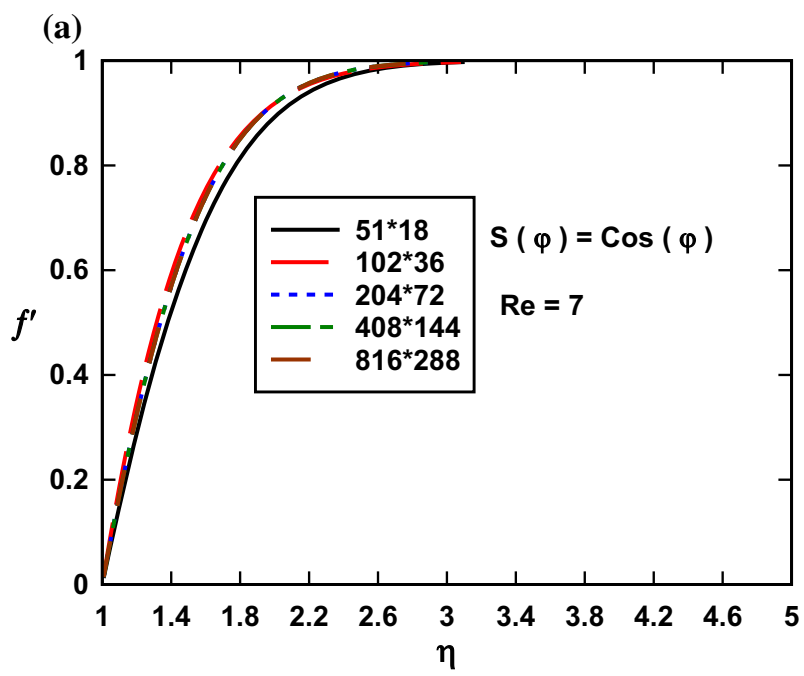

(b)

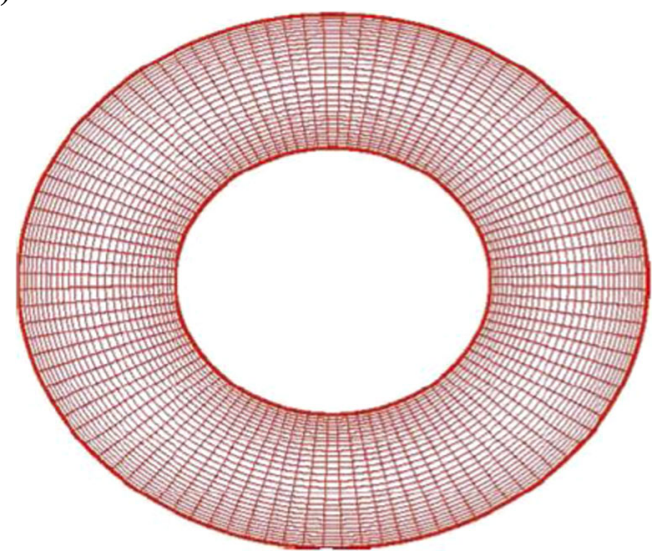

Fig. 2 a Radial distribution of $f^{\prime}(\eta, \varphi)$ for different mesh sizes at $\lambda=0, R e=7.0, S(\varphi)=\cos (\varphi)$, b typical grid system 


$$
\begin{aligned}
& \varepsilon \cdot\left(\eta f^{\prime \prime \prime}+f^{\prime \prime}+\frac{1}{4 \eta} \frac{\partial^{2} f^{\prime}}{\partial \varphi^{2}}\right)+\operatorname{Re}\left[1+f f^{\prime \prime}-\left(f^{\prime}\right)^{2}\right]+\varepsilon^{2} \cdot \lambda\left[1-f^{\prime}\right]=0, \\
& P-P_{0}=-\frac{1}{2 \varepsilon^{2}}\left(\frac{f^{2}}{\eta}\right)-\frac{1}{\varepsilon}\left(\frac{f^{\prime}}{\operatorname{Re}}\right)-\frac{1}{4 \varepsilon R e} \int_{1}^{\eta} \frac{1}{\eta^{2}} \frac{\partial^{2} f}{\partial \varphi^{2}} \mathrm{~d} \eta+\frac{1}{\varepsilon} \frac{\lambda}{\operatorname{Re}} \int_{1}^{\eta} \frac{f}{\eta} \mathrm{d} \eta \\
& \quad-2\left[\frac{1}{\varepsilon^{2}}+\frac{\lambda}{\operatorname{Re}}\right]\left(\frac{z}{a}\right)^{2}, \\
& \eta \theta^{\prime \prime}+\theta^{\prime}+\frac{1}{4 \eta} \frac{\partial^{2} \theta}{\partial \varphi^{2}}+\operatorname{Re} \cdot \operatorname{Pr}\left(f \theta^{\prime}\right)=0 .
\end{aligned}
$$

\subsection{Shear Stress and Nusselt Number}

The shear stress at the cylinder surface is given by Cunning et al. (1998) and Alizadeh et al. $(2016 b, c)$

$$
\sigma=\mu\left[\frac{\partial w}{\partial r}\right]_{r=a},
$$

where $\mu$ is the fluid viscosity. Using the transformations introduced in Eq. (11), the shear stress on the cylinder surface for semi-similar solutions becomes

$$
\sigma=\mu \frac{2}{a}\left[2 \bar{k} z f^{\prime \prime}(1, \varphi)\right] \Rightarrow \frac{\sigma a}{4 \mu \bar{k} z}=f^{\prime \prime}(1, \varphi) .
$$

The local heat transfer coefficient and rate of heat transfer for defined wall temperature are defined as

$$
h=\frac{q_{\mathrm{w}}}{T_{w}-T_{\infty}}=\frac{-k\left(\frac{\partial T}{\partial r}\right)_{r=a}}{T_{w}-T_{\infty}}=-\frac{2 k}{a} \frac{\partial \theta(1, \varphi)}{\partial \eta}
$$

\begin{tabular}{|c|c|c|c|c|c|c|}
\hline \multirow[t]{2}{*}{$R e$} & \multicolumn{2}{|c|}{$P r=0.2$} & \multicolumn{2}{|c|}{$P r=2.0$} & \multicolumn{2}{|l|}{$P r=20$} \\
\hline & $\begin{array}{l}\text { Present } \\
\text { work }\end{array}$ & Wang (1974) & $\begin{array}{l}\text { Present } \\
\text { work }\end{array}$ & Wang (1974) & $\begin{array}{l}\text { Present } \\
\text { work }\end{array}$ & Wang (1974) \\
\hline 1.0 & 0.2569 & 0.2572 & 1.066 & 1.065 & 3.523 & 3.521 \\
\hline 10.0 & 0.6160 & 0.6164 & 3.033 & 3.035 & 10.73 & 10.77 \\
\hline 100 & 1.775 & 1.779 & 9.259 & 9.262 & 33.64 & 33.72 \\
\hline
\end{tabular}

and

$$
q_{\mathrm{w}}=-\frac{2 k}{a} \frac{\partial \theta(1, \varphi)}{\partial \eta}\left(T_{w}-T_{\infty}\right)
$$

Table 1 Comparison between the Nusselt numbers in the current work and those of Wang (1974) for $S(\varphi)=0$ and $\lambda=0$

Table 2 Default values of the simulation parameters

\begin{tabular}{llllllll}
\hline $\begin{array}{l}\text { Simulations } \\
\text { parameters }\end{array}$ & $\eta$ & $\varepsilon$ & $\varphi$ & $\lambda$ & $\operatorname{Re}$ & $\operatorname{Pr}$ & Transpiration rate \\
\hline & 1.45 & 0.9 & $72^{\circ}$ & $\lambda=1.0$ & 10 & 1.0 & $S(\varphi)=\cos (\varphi)$ \\
\hline
\end{tabular}


(a)

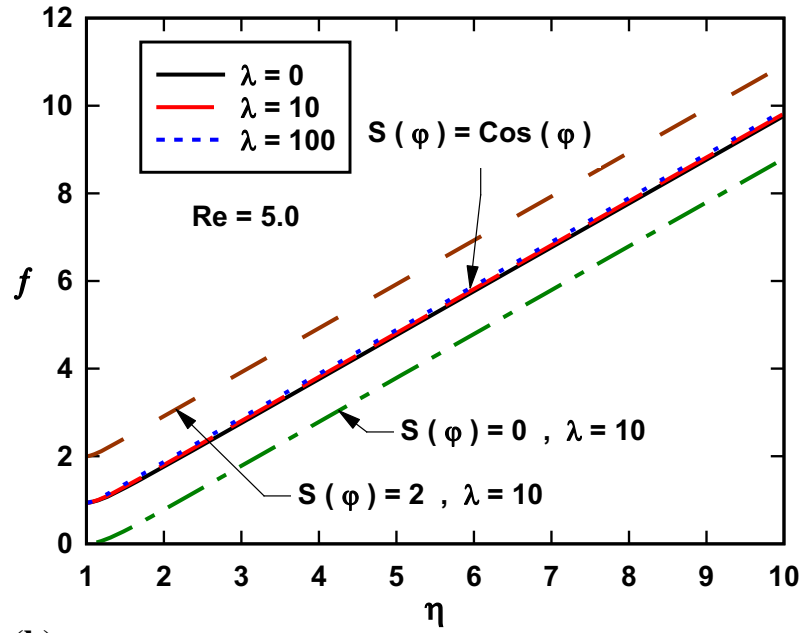

(b)

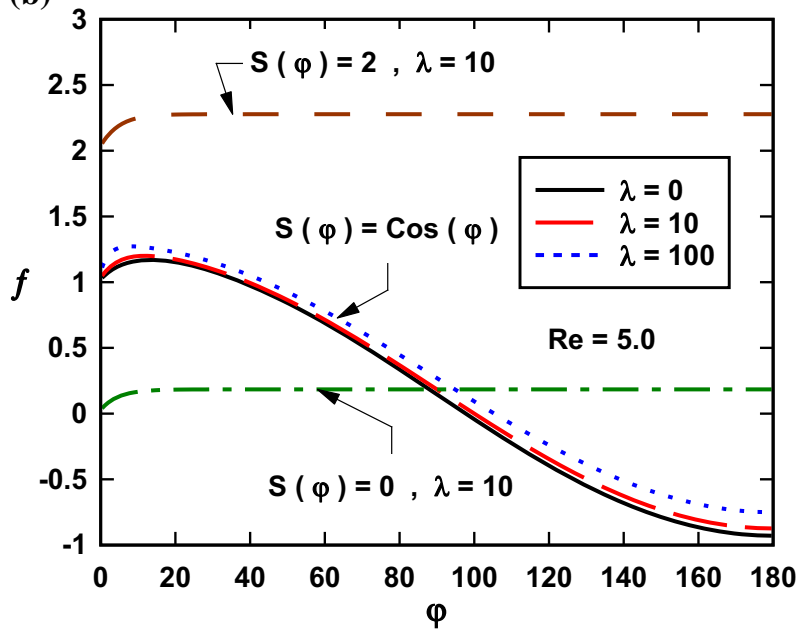

Fig. 3 Variations of $f(\eta, \varphi)$ with $\mathbf{a} \eta$ and $\mathbf{b} \varphi$ at $\operatorname{Re}=5.0, S(\varphi)=\cos (\varphi)$ and for different values of $\lambda$

Therefore, Nusselt number takes the form of

$$
N u=\frac{h a}{2 k}=-\theta^{\prime}(1, \varphi) .
$$

\subsection{Grid Independency and Validation}

To ensure the grid independency of the developed numerical solution, variations of the $\eta$ with $f^{\prime \prime}(\eta, \varphi)$ on the cylinder were examined with $(\eta, \varphi)$ mesh sizes of $51 \times 18,102 \times 36$, $204 \times 72,408 \times 144$ and $816 \times 288$. Figure 2 a shows no considerable changes in the $f^{\prime \prime}(\eta, \varphi)$ for $(\eta, \varphi)$ mesh sizes of $(204 \times 72),(408 \times 144)$ and $(816 \times 288)$. Thus, a $(408 \times 144)$ grid in $\eta-\varphi$-directions was applied to the computational domain. A non-uniform grid was employed in $\eta$-direction to capture the sharp gradients around the external surface of the cylinder, while 
(a)

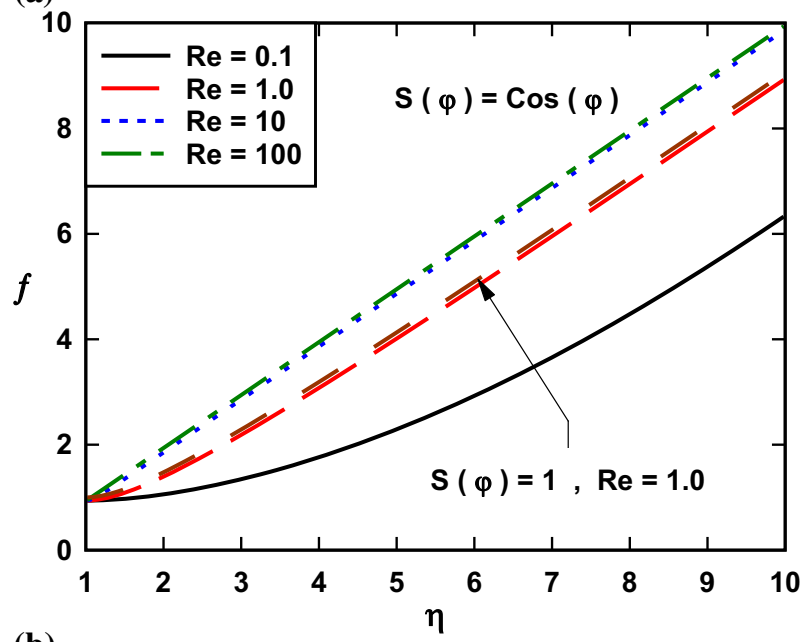

(b)

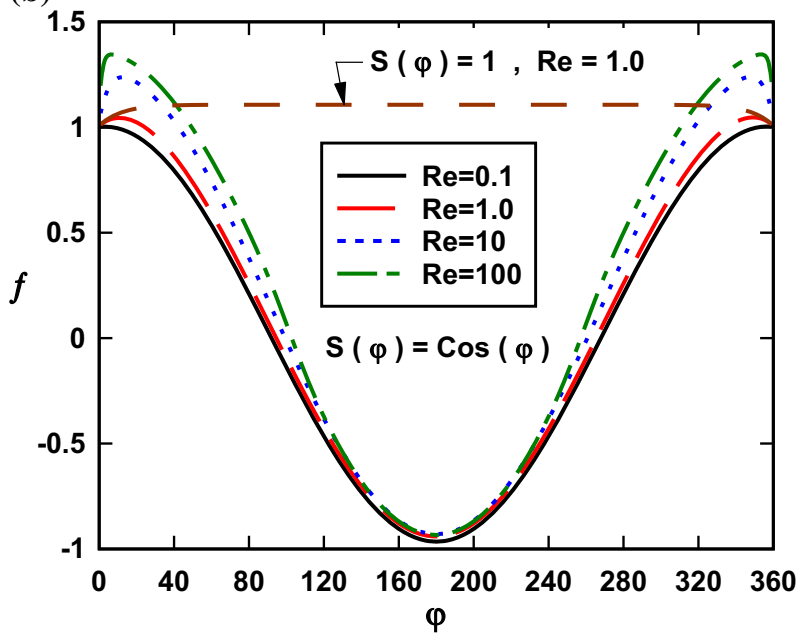

Fig. 4 Variations of $f(\eta, \varphi)$ with $\mathbf{a} \eta$ and $\mathbf{b} \varphi$ at $\lambda=10, S(\varphi)=\cos (\varphi)$ and for different values of Reynolds numbers

a uniform mesh was implemented in the $\varphi$-direction. The computational domain extends over $\varphi_{\max }=360^{\circ}$ and $\eta_{\max }=15$, where $\eta_{\max }$ corresponds to $\eta \rightarrow_{\infty}$, which lies very well outside the momentum and thermal boundary layers. Figure $2 \mathrm{~b}$ illustrates the computational mesh used in the current study. A convergence criterion based on the relative difference between two consecutive iterations was employed. When this difference was less than $10^{-7}$, the solution was assumed to have converged and hence the iterative process was terminated. On the basis of the implemented numerical scheme the numerical error is of $O(\Delta \eta)^{2}$ (Thomas 2013). In order to examine the validity of the solutions developed in Sects. 2.2 and 2.3, the Nusselt number calculated through Eq. (31) was compared with those obtained from the literature for flows over cylinders with no transpiration and infinitely large permeability. The outcomes are shown in Table 1, and the close agreement between the two sets of Nusselt number confirms the validity of the conducted numerical simulations. 


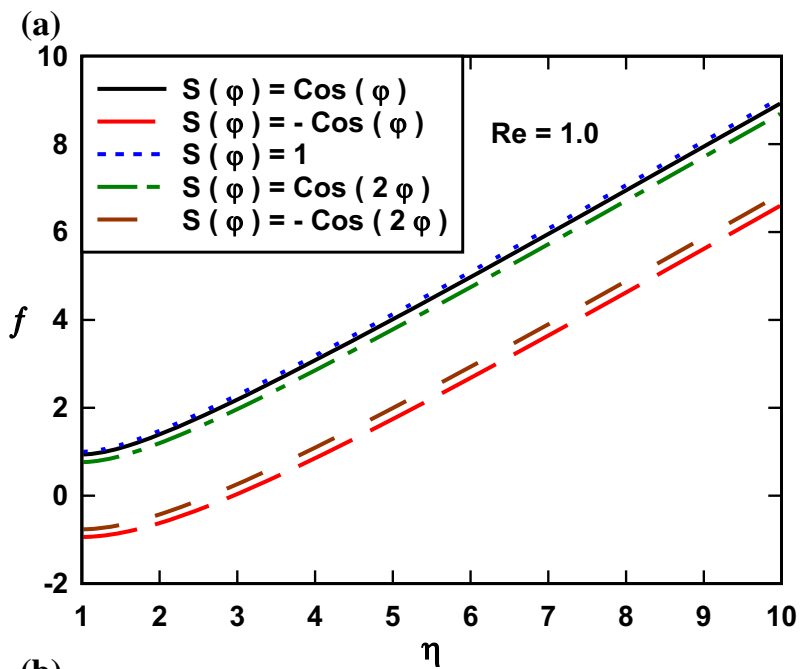

(b)

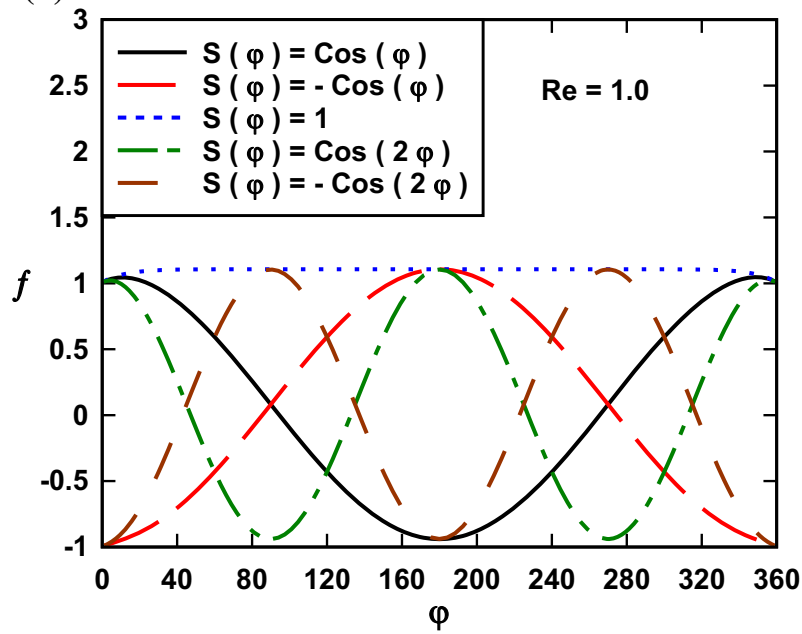

Fig. 5 Variations of $f(\eta, \varphi)$ with $\mathbf{a} \eta$ and $\mathbf{b} \varphi$ at $R e=1.0$ and for different transpiration rate functions

\section{Results and Discussion}

The results presented in this section are on the basis of the default values of parameters presented in Table 2. Any changes to these values have been explicitly shown in the figures and tables. Three types of transpiration functions have been employed. These include $S=0$, $S=$ const., and $S=\cos (\varphi)$, which have been further restated in the figures and tables.

\subsection{Flow Velocity Field}

The radial and circumferential variations of the dimensionless radial velocity $(f)$ are shown in Fig. 3 for different values of the reciprocal of Darcy number $\lambda$ and transpiration functions. As expected, this figure confirms that the form of transpiration function heavily affects the 

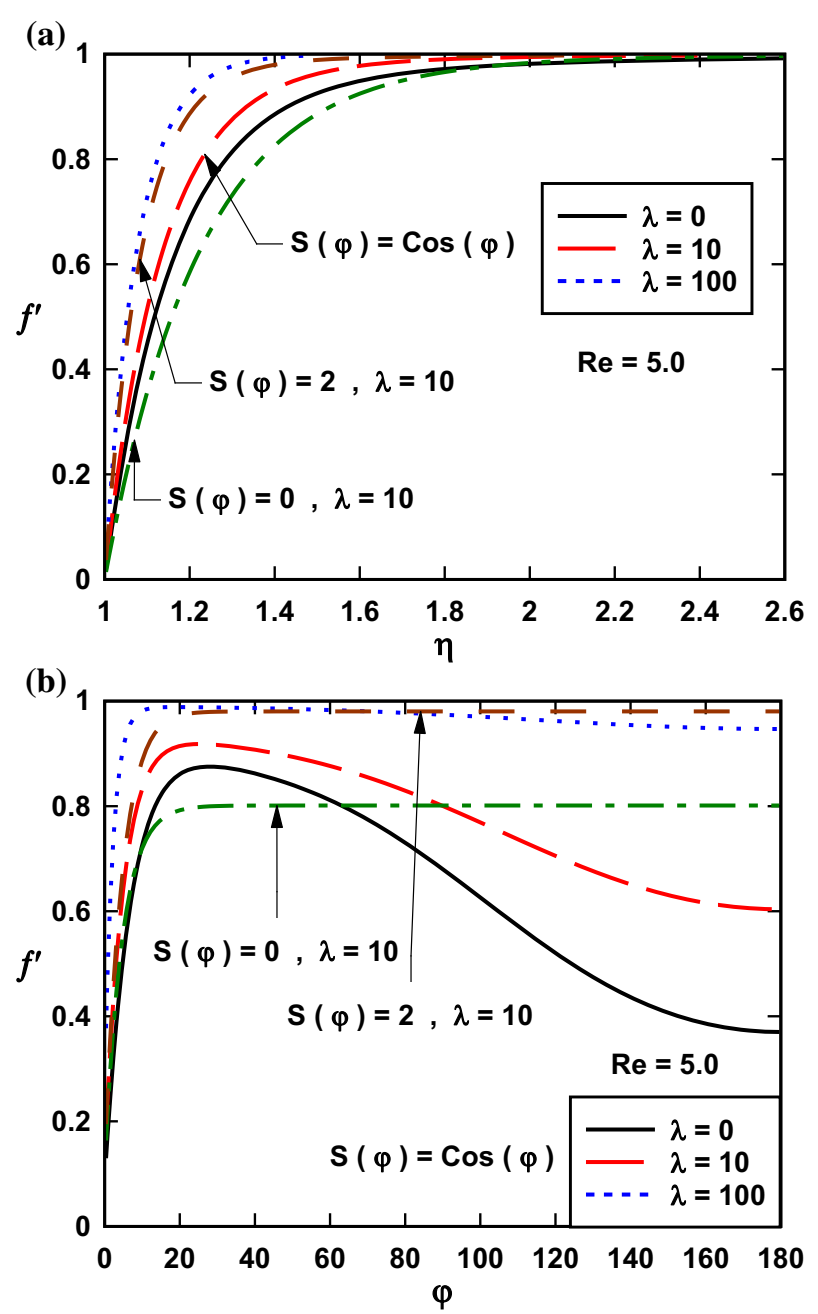

Fig. 6 Variations $f^{\prime}(\eta, \varphi)$ with $\mathbf{a} \eta$ and $\mathbf{b} \varphi$ at $\operatorname{Re}=5.0, S(\varphi)=\cos (\varphi)$ and for different values of $\lambda$

variation of $f$ in the radial and circumferential directions. Figure 3 further indicates that large changes in the $\lambda$ have relatively small influences upon $f$. It should be noted that the external flow Reynolds number has been kept constant in Fig. 3 and hence the freestream strain rate is unchanged. Figure 3 also shows that for zero transpiration the qualitative behaviour of the non-dimensional velocity in the radial direction is in qualitative agreement with that of transversal dimensionless velocity in Wu et al. (2005) and Kokubun and Fachini (2011). This is to be expected as the radial velocity in the cylindrical configuration shown in Fig. 1 is analogous to the transversal velocity in the flow over a flat porous insert investigated in $\mathrm{Wu}$ et al. (2005) and Kokubun and Fachini (2011). Figure 4 depicts the influences of freestream Reynolds number (defined as $R e=\frac{k a^{2}}{2 v}$ ) on the non-dimensional velocity $f$. Reynolds number appears to be particularly influential on the radial distribution of $f$, in which increasing the Reynolds number from 0.1 to 10 can almost double the value of $f$. Once again, the strong effects of transpiration rate are evident in Fig. 4. It is interesting 


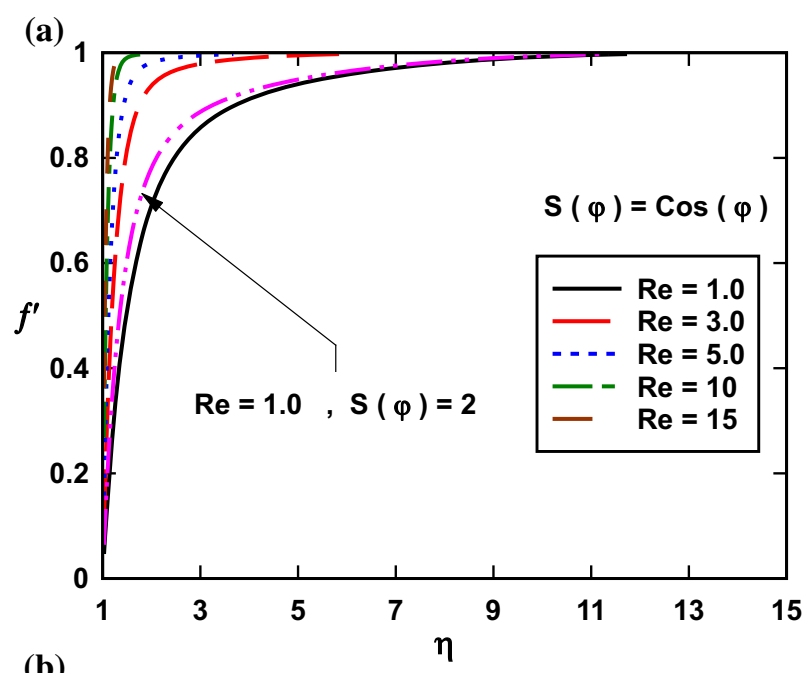

(b)

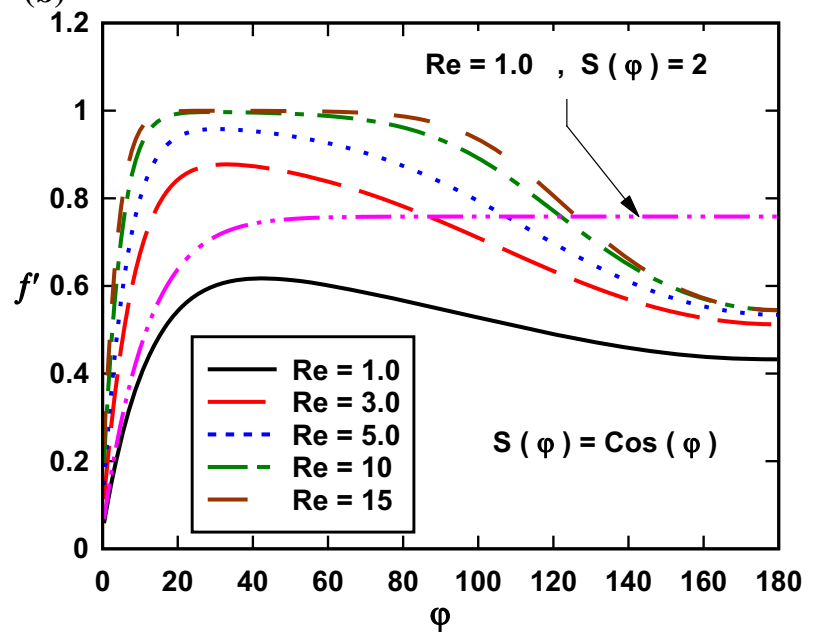

Fig. 7 Variations of $f^{\prime}(\eta, \varphi)$ with $\mathbf{a} \eta$ and $\mathbf{b} \varphi$ at $\lambda=10, S(\varphi)=\cos (\varphi)$ and for different values of Reynolds numbers

to note that the radial distribution of $f$ is only quantitatively influenced by the changes in the transpiration function. However, the circumferential distribution of $f$ is heavily affected by the transpiration function in both qualitative and quantitative manners, see Fig. 4b. To further elaborate on the effects of transpiration function, Fig. 5 shows the distributions of $f(\eta, \varphi)$ for different functional forms of the transpiration rate. It is clear from Fig. 5a that the qualitative trend in the radial distribution of $f(\eta, \varphi)$ is simple and remains indifferent to changes in the transpiration rate. However, this is significantly different for the circumferential distribution of $f$ shown in Fig. $5 \mathrm{~b}$ in which the non-dimensional velocity closely follows the transpiration rate. This is due to the kinematic balance of the velocity components induced by the transpiration and external flow around the stagnation point.

The behaviours of the dimensionless axial velocity, $f^{\prime}(\eta, \varphi)$, are investigated in Figs. 6 , 7 and 8 . Figure 6 examines the effects of permeability of the porous medium on $f^{\prime}(\eta, \varphi)$. 

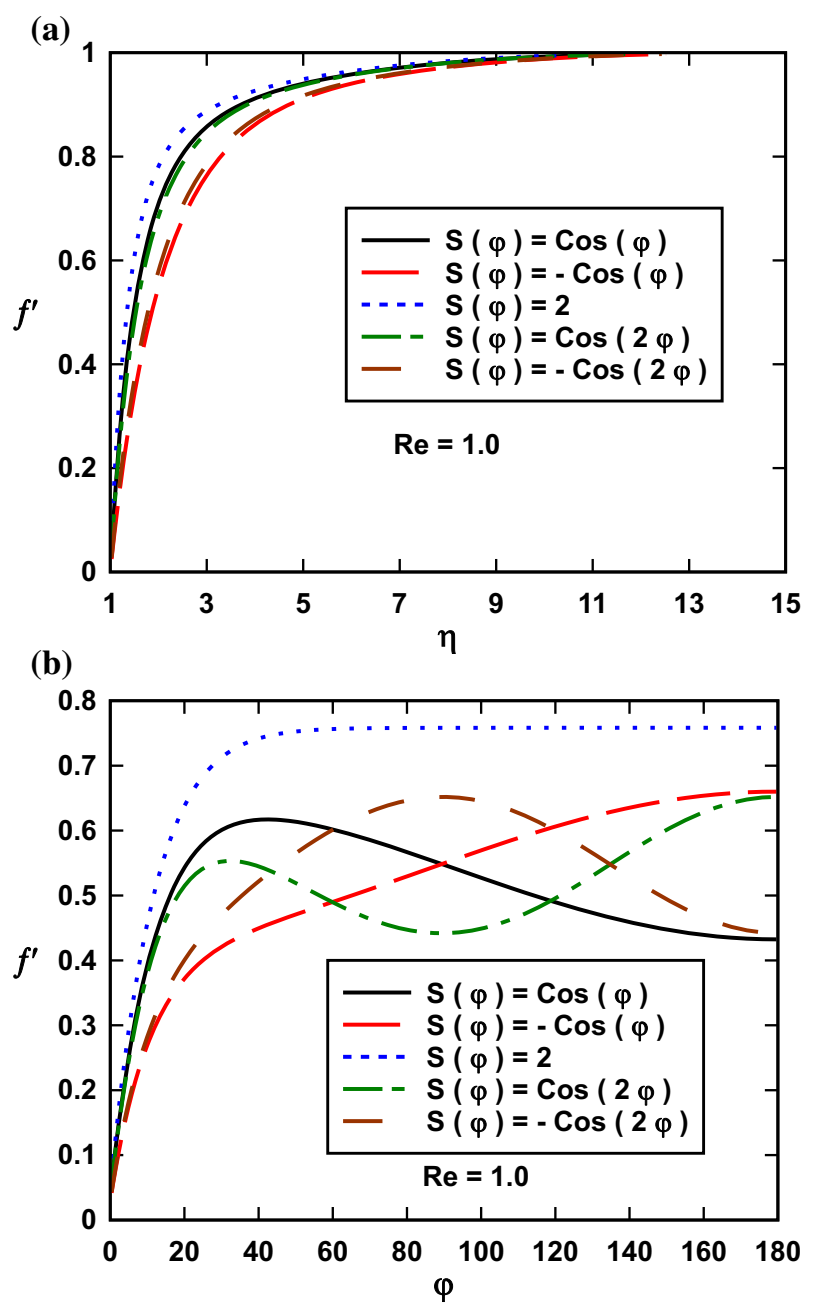

Fig. 8 Variations of $f^{\prime}(\eta, \varphi)$ in terms of $\mathbf{a} \eta$ and $\mathbf{b} \varphi$ at $\lambda=10, R e=1.0$ and for different transpiration rate functions

Part a of this figure shows that at large enough values of dimensionless radius and for all values of $\lambda$ and transpiration rate, the values of $f^{\prime}$ approach the same value. However, the circumferential distribution of $f^{\prime}$, shown in Fig. 6b, features a strong dependency upon the transpiration rate. For zero and constant transpiration rate this includes a sharp increase with respect to $\varphi$ followed by an asymptotic approach towards a constant value. Introduction of non-uniform transpiration complicates this behaviour in which the initial sharp increase in $f^{\prime}$ is followed by a strongly permeability depend drop. As a general trend, Fig. 6 shows that by increasing $\lambda$, the numerical value of $f^{\prime}$ increases. This is in keeping with the findings of $\mathrm{Wu}$ et al. (2005) for the isothermal flow on a flat porous insert and is related to the increases in the thickness of momentum at lower permeability of the porous medium. Figure 7 depicts the substantial effects of Reynolds number on $f^{\prime}$. For very small values of Reynolds number $(R e=1.0)$, the radial distribution of $f^{\prime}$ is almost perfectly linear, see Fig. 7a. In the circumferential direction, it grows from zero at $\varphi=0$ and reaches a constant value at 

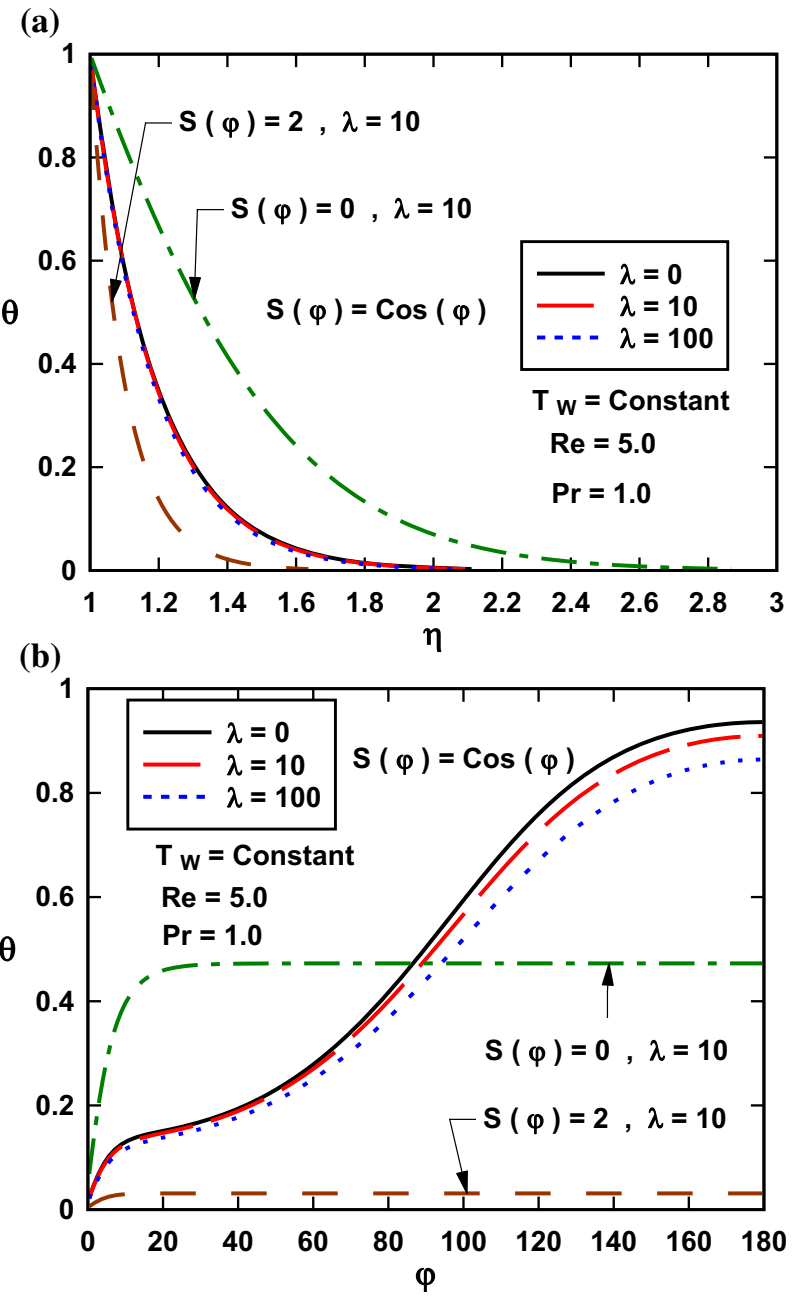

Fig. 9 Variations of $\theta(\eta, \varphi)$ with $\mathbf{a} \eta$ and $\mathbf{b} \varphi$ at $\operatorname{Pr}=1.0, \operatorname{Re}=5.0, S(\varphi)=\cos (\varphi)$ and for different values of $\lambda$

higher value of $\varphi$. These behaviours are only slightly modified at $R e=3.0$. However, for higher values of Reynolds number the initial increase in $f^{\prime}$ with respect to $\eta$ becomes much sharper, while the rate of increase slows down at larger values of $\eta$. For the non-uniform transpiration function and in the circumferential direction $f^{\prime}$ can further include negative growth. This is particularly the case at high Reynolds numbers, which tend to magnify the effects of transpiration rates. Figure 8 illustrates the dependency of $f^{\prime}$ on the functional form of the transpiration rate. Similar to that discussed in Fig. 5, the influences of transpiration rate are more noticeable in the circumferential distribution of $f^{\prime}$. Figure 8a shows that the non-dimensional axial velocity is nearly zero in the vicinity of the stagnation point. However, for small values of $\varphi$ and for all investigated transpiration functions it increases rapidly in the angular direction. Further developments of the non-dimensional axial velocity follow the functional form of the transpiration rate. 

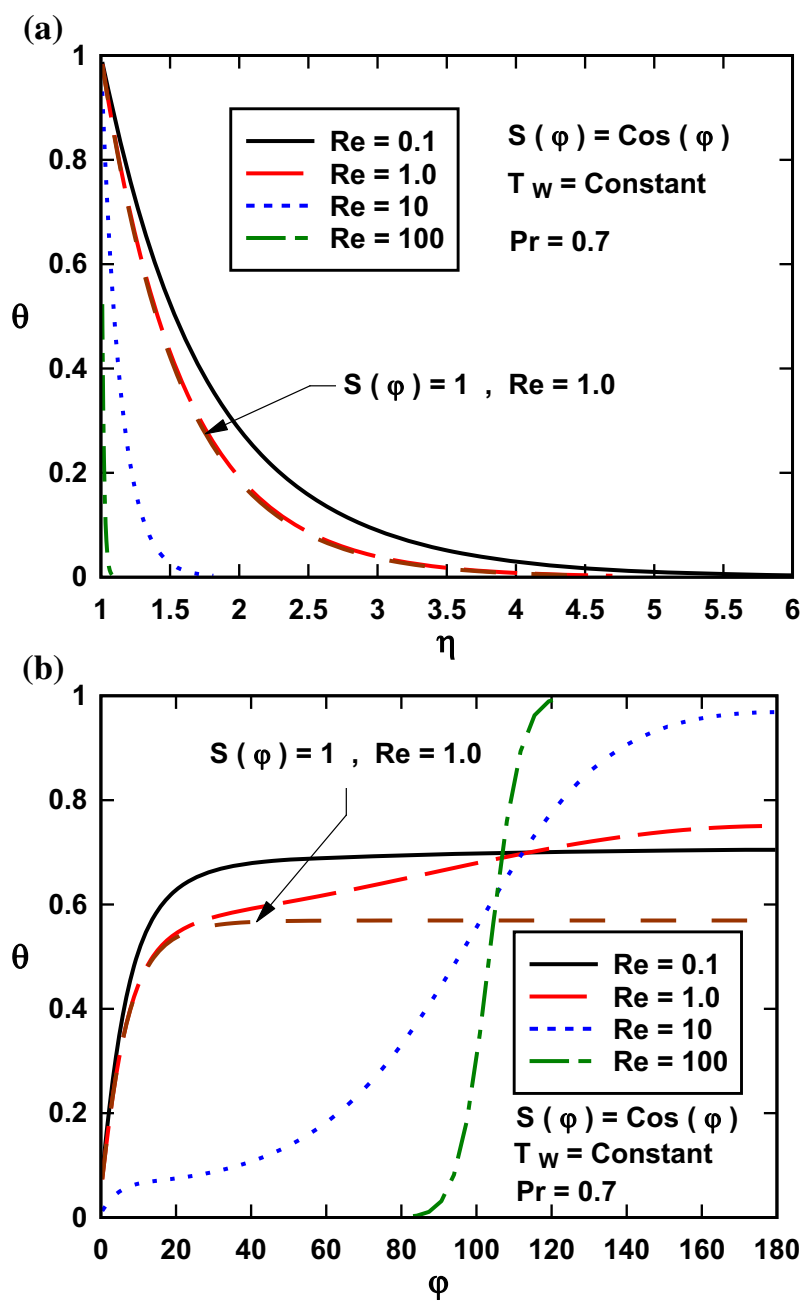

Fig. 10 Variations of $\theta(\eta, \varphi)$ with $\mathbf{a} \eta$ and $\mathbf{b} \varphi$ at $\operatorname{Pr}=0.7, S(\varphi)=\cos (\varphi)$ and for different values of Reynolds number

\subsection{Flow Temperature Field}

Figure 9 shows the radial and circumferential distribution of the non-dimensional temperature, $\theta(\eta, \varphi)$, for different values of $\lambda$ and three types of transpiration rate. Similar to that discussed for the velocity field, the influences of permeability upon the radial distribution of flow temperature appear to be minor, see Fig. 9a. However, the transpiration rate significantly alters the temperature distribution. The almost uniform circumferential temperature distribution in the cases of zero and uniform transpiration and the strong variations of $\theta$ with $\varphi$ for non-uniform transpiration are very noticeable in Fig. 9b. Variation of non-dimensional temperature with respect to the dimensionless radius, $\eta$, gives an indication of the thickness of the thermal boundary layer on the surface of cylinder. Figure $9 \mathrm{a}$ shows that with no transpiration the thermal boundary layer is relatively thick as nonzero values of $\theta$ prevail 


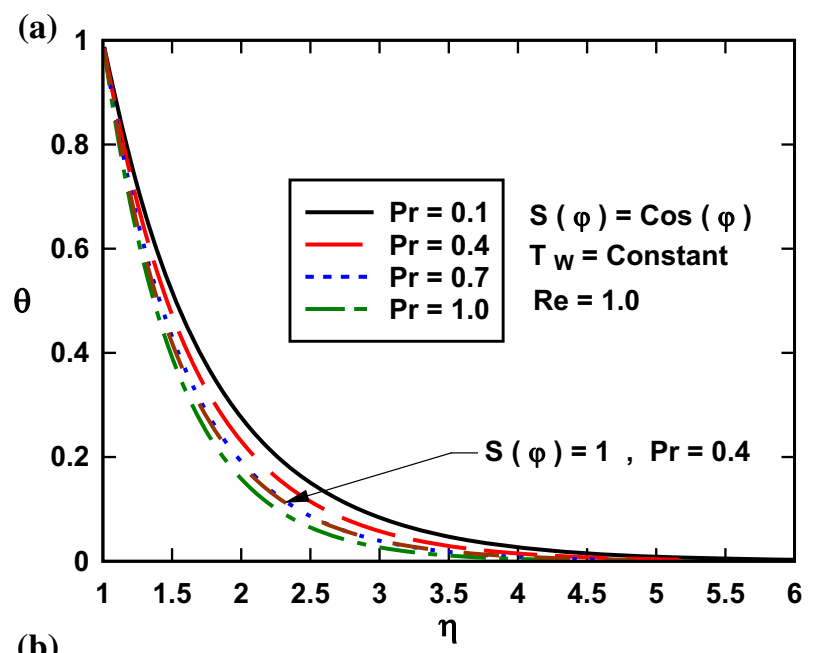

(b)

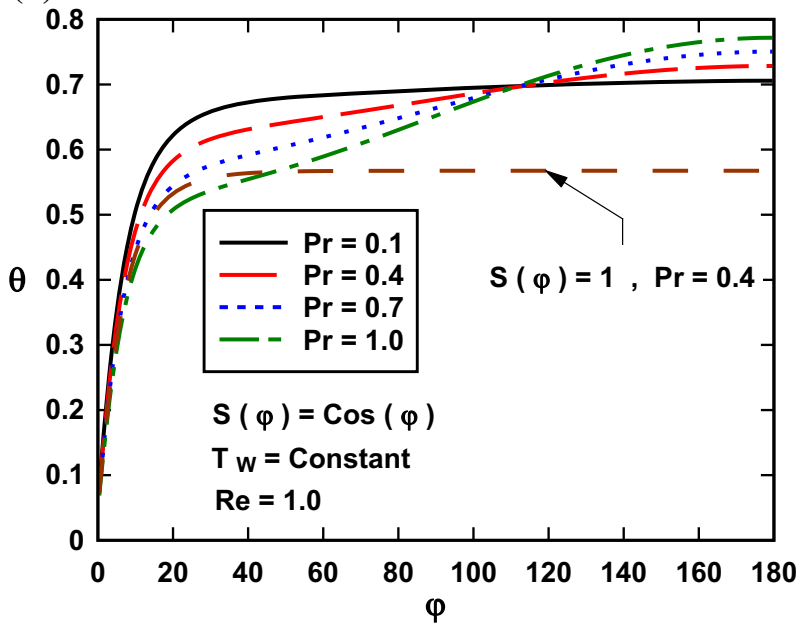

Fig. 11 Variations of $\theta(\eta, \varphi)$ with $\mathbf{a} \eta$ and $\mathbf{b} \varphi$ at $\operatorname{Re}=1.0, S(\varphi)=\cos (\varphi)$ and for different values of Prandtl number

to $\eta \cong 2$.6. Yet, the addition of transpiration (suction) causes a significant decrease in the thickness of the thermal boundary layer. The strongest reduction is by constant transpiration, in which the value of $\theta$ reaches zero at $\eta \cong 1.5$.

Figure 10 depicts the effects of Reynolds number upon the flow temperature field. Figure 10a implies that increasing the Reynolds number of the external flow substantially decreases the thickness of the thermal boundary layer and limits the heat transfer to a narrow layer of fluid around the cylinder. This implied indicated by the rapid drop of $\theta$ at higher Reynolds numbers. It means the fluid temperature reaches that of the freestream and therefore the thermal boundary layer terminates at smaller values of $\eta$. The interpretation of the results in Fig. 10b is quite involved. In general, this figure indicates that by increasing the Reynolds number a larger fraction of the cylinder circumference becomes exposed to lower values of $\theta$. In explaining this behaviour, it should be first noted that, as shown in Table 2, the results in Fig. 10b correspond to $\eta=1.45$. Hence, $\theta=0$ in this figure implies that 

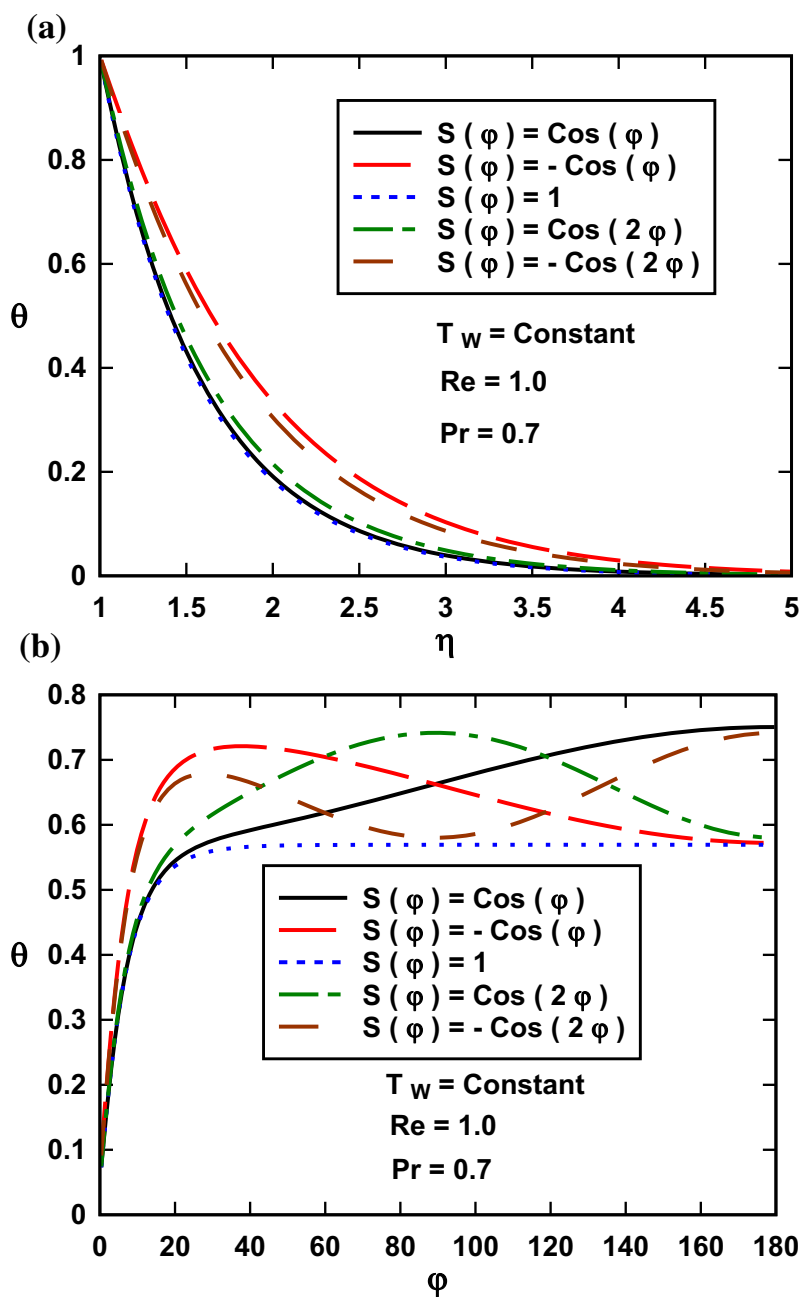

Fig. 12 Variation of $\theta(\eta, \varphi)$ in terms of $\mathbf{a} \eta$ and $\mathbf{b} \varphi$, at $\operatorname{Pr}=0.7, \operatorname{Re}=1.0$ and for different transpiration rate functions

thickness of the thermal boundary layer is smaller than $\eta=0.45$. Considering this, Fig. $10 \mathrm{~b}$ clearly reflects the thickness reduction of the thermal boundary layer at higher Reynolds numbers. The influences of the fluid Prandtl number on the temperature field are investigated in Fig. 11. As expected, by increasing Prandtl number the heat transfer from the cylinder intensifies. This results in smaller temperature difference between the fluid and the surface of the cylinder, which in turn reduces the value of $\theta$. A similar qualitative trend was detected by Bhattacharyya and Layek (2011) in their analysis of flow over a flat porous medium with uniform transpiration. The impacts of transpiration rate on the temperature field are illustrated in Fig. 12. Variations of the temperature field with respect to changes in the functional form of the transpiration rate are further investigated in this figure. An interesting feature observed in Fig. 12 is the temperature differences induced by blowing and suction. The positive sign of transpiration function refers to suction, while the negative sign indicates blowing. 

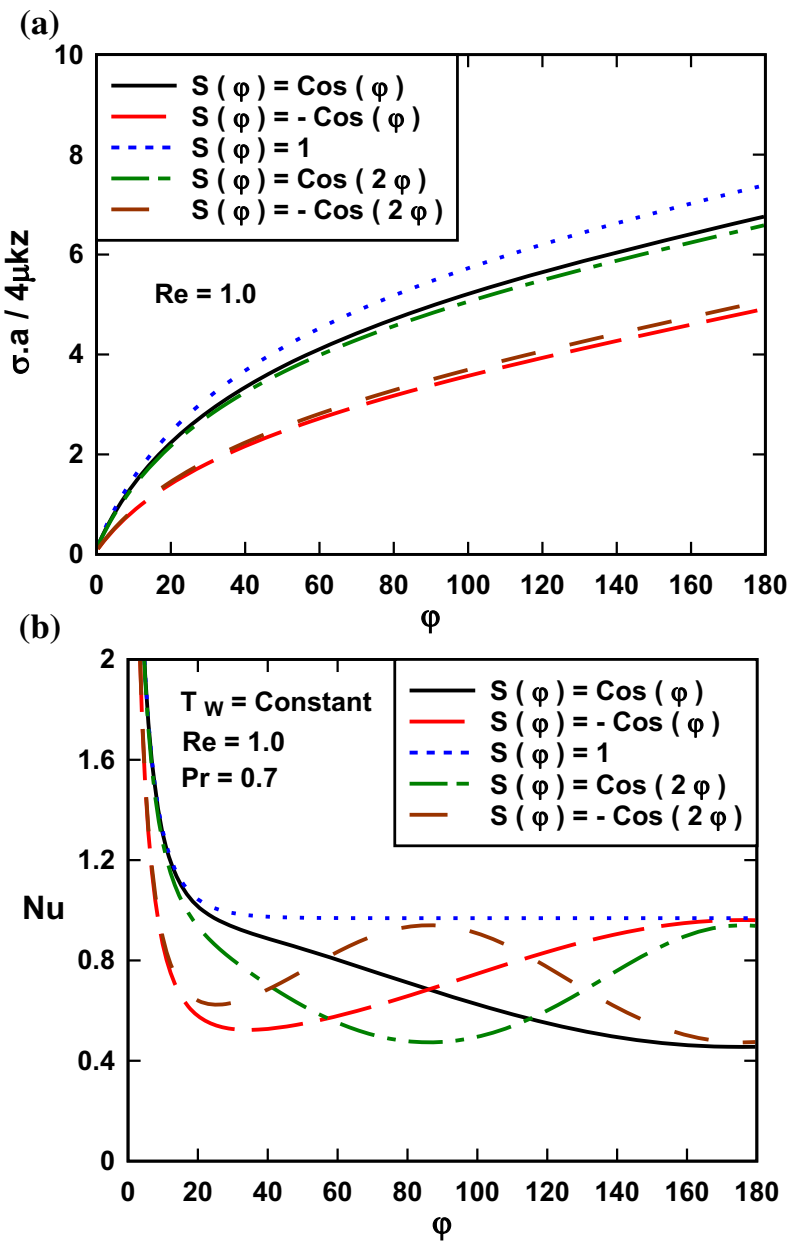

Fig. 13 Effects of variations in the transpiration rate function a on the shear stress, $\mathbf{b}$ on the local Nusselt number

Figure 12a clearly shows that blowing results in larger non-dimensional temperatures, which indicates greater temperature differences between the fluid and the surface of the cylinder. The reason for this behaviour can be attributed to the thickness of the boundary layer and its adverse effect on the heat transfer rate. Surface suction of the fluid reduces the thickness of the boundary layer. This strengthens the heat convection and reduces the temperature difference between the surface of the cylinder and the fluid. Blowing, however, makes the hydrodynamic and thermal boundary layers thicker and therefore impedes heat transfer by convection and results in increasing the non-dimensional temperature.

\subsection{Nusselt Number, Shear Stress and Pressure Drop}

Stagnation-point flows often generate significant shear stress on the solid surface (Alizadeh et al. 2016a, b), which contributes to the erosion of the system and hence should be evaluated carefully. Figure 13a shows the circumferential distribution of non-dimensional shear stress. 
Table 3 Effects of Reynolds number and $\lambda$ on the average shear stress $\left(\sigma_{m} \cdot a / 4 \mu \bar{k} z\right)$ for $S(\varphi)=\cos (\varphi)$ and $\operatorname{Pr}=1.0$

\begin{tabular}{llll}
\hline$R e$ & $\sigma_{m} \cdot a / 4 \mu \bar{k} z$ & $\lambda$ & $\sigma_{m} \cdot a / 4 \mu \bar{k} z$ \\
\hline 0.01 & 0.09413 & 0 & 1.43428 \\
0.1 & 0.21868 & 1.0 & 2.05037 \\
1.0 & 1.43428 & 10 & 5.64413 \\
10 & 10.65943 & 50 & 13.52675 \\
100 & 38.12034 & 100 & 18.75878 \\
\hline
\end{tabular}

(a)
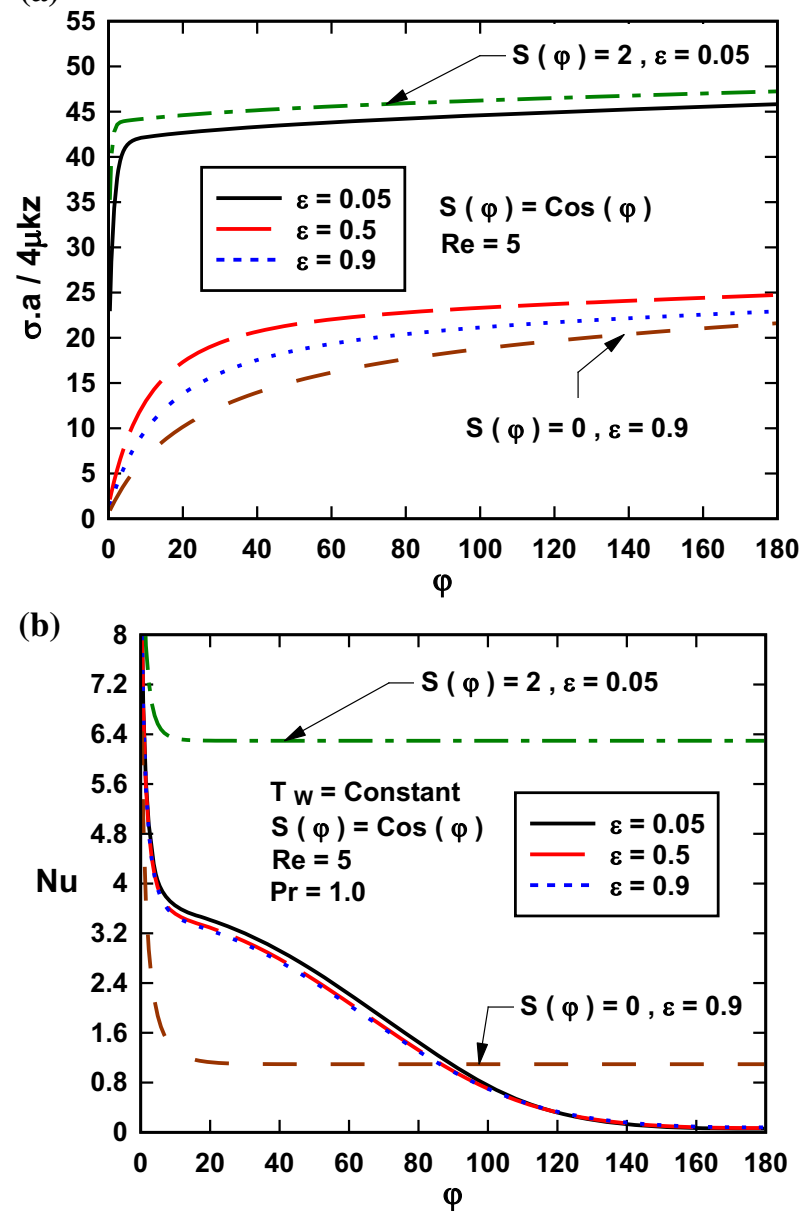

Fig. 14 Effects of variations in the porosity on $\mathbf{a}$ the shear stress, $\mathbf{b}$ the local Nusselt number

Expectedly, there is no shear stress at the stagnation point, and as the value of $\varphi$ increases and flow gains momentum, the shear stress increases monotonically. The rate of this increase appears to be dependent upon the functional form of transpiration, and for the cases investigated in Fig. 13a the maximum growth rate corresponds to the uniform transpiration. The average values of the shear stress around the cylinder are presented in Table 3 for varying 
Table 4 Effects of Prandtl number and $\lambda$ on the average Nusselt number $(\mathrm{Nu})$ for $S(\varphi)=0, R e=1.0$

\begin{tabular}{llll}
\hline $\operatorname{Pr}$ & $N u_{m}$ & $\lambda$ & $N u_{m}$ \\
\hline 0.1 & 3.59774 & 0 & 3.84219 \\
0.4 & 3.74114 & 1.0 & 3.84888 \\
0.7 & 3.84888 & 10 & 3.88851 \\
1.0 & 3.93788 & 50 & 3.95263 \\
10 & 5.07670 & 100 & 3.98287 \\
\hline
\end{tabular}

\begin{tabular}{llll}
\hline$P r$ & $N u_{m}$ & $\lambda$ & $N u_{m}$ \\
\hline 0.1 & 3.89342 & 0 & 6.19832 \\
0.4 & 5.02176 & 1.0 & 6.20146 \\
0.7 & 6.20146 & 10 & 6.22214 \\
1.0 & 7.41599 & 50 & 6.26340 \\
10 & 44.32687 & 100 & 6.28660 \\
\hline
\end{tabular}

\begin{tabular}{llll}
\cline { 2 - 4 } Pr & $N u_{m}$ & $\lambda$ & $N u_{m}$ \\
\hline 0.1 & 3.59124 & 0 & 4.12700 \\
0.4 & 3.76584 & 1.0 & 4.13656 \\
0.7 & 3.94454 & 10 & 4.16776 \\
1.0 & 4.126997 & 50 & 4.19767 \\
10 & 10.52329 & 100 & 4.20916 \\
\hline
\end{tabular}

Table 5 Effects of Prandtl number and $\lambda$ on the average Nusselt number $\left(\mathrm{Nu}_{m}\right)$ for $S(\varphi)=1, \operatorname{Re}=1.0$
Table 6 Effects of Prandtl number and $\lambda$ on the average Nusselt number $\left(N u_{m}\right)$ for

$\operatorname{Re}=1.0$

Reynolds number and $\lambda$. The substantial growth of the average shear stress with respect to Reynolds number and $\lambda$ is evident in this table. Figure $13 \mathrm{~b}$ shows the circumferential distribution of the local Nusselt number. In agreement with that observed in other heat transferring stagnation-point flows (Saleh and Rahimi 2004 and Alizadeh et al. 2016b,c), the numerical value of Nusselt number at the stagnation point is very large. This value drops quickly at higher values of $\varphi$ as the boundary layers starts to develop. Figure 13b shows that uniform transpiration results in constant Nusselt number for $\varphi \geq 20^{\circ}$. However, non-uniform transpirations lead to significant fluctuations in the distribution of Nusselt number in the circumferential direction.

Figure 14 depicts the influences of porosity of the porous medium upon the shear stress and averaged Nusselt number. As shown in this figure the dimensionless shear stress on the surface of the cylinder is largely affected by the variations in the porosity. Figure 14a shows that increasing porosity results in significant reduction in the shear stress. Nonetheless, this is clearly not the case for Nusselt number, which appears to be almost independent of the porosity (see Fig. 14b). Although not shown in here, the temperature field also features a very weak dependency on the porosity. In interpreting these results, it is important to note that the values of permeability, represented by $\lambda$, are considered constant in Fig. 14. To further investigate the effects of transpiration, Tables 4, 5 and 6 present the numerical values of the Nusselt number averaged around the circumference of the cylinder $\left(N u_{m}\right)$ for three different types of transpiration. Averaged Nusselt numbers are reported against Prandtl number and $\lambda$. A comparison of the reported values of the averaged Nusselt number in these 

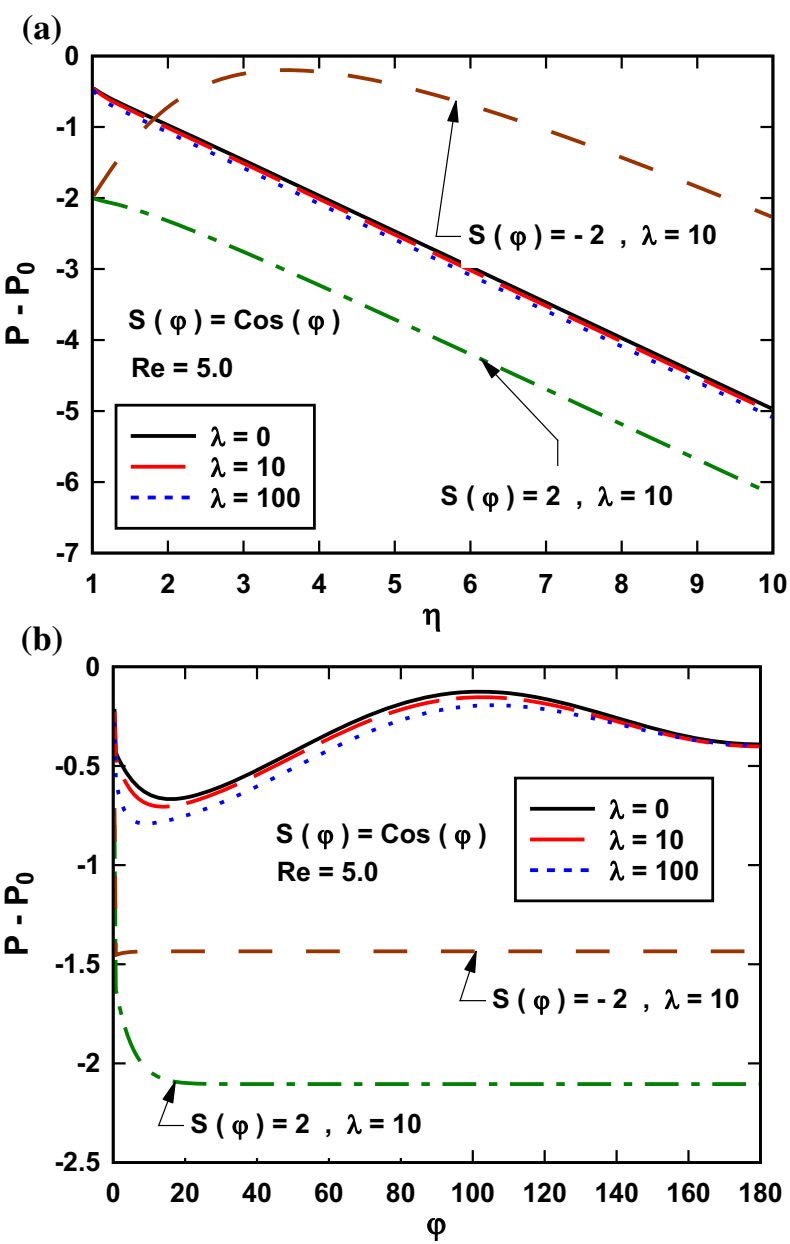

Fig. 15 Variations of $P-P_{0}$ with $\mathbf{a} \eta$ and $\mathbf{b} \varphi$ at $\operatorname{Re}=5.0, S(\varphi)=\cos (\varphi)$ and for different values of $\lambda$

tables reveals the major effects of the transpiration function. At low values of Prandtl number the averaged Nusselt number for all considered transpiration functions is more and less the same. However, as Prandtl number increases the rate of increase in the averaged Nusselt number becomes significantly dependent upon the type of transpiration. This is such that under no transpiration $\mathrm{Nu}_{m}$ grows for less than 50\% for the investigated range of Prandtl number (Table 3). However, $N u_{m}$ increases by three times under the non-uniform (Table 6) and by more than ten times under uniform transpiration (Table 5). This great dependency of $N u_{m}$ upon the transpiration function is in agreement with the results discussed in Sects. 3.1 and 3.2 about the influences of transpiration function on the velocity and temperature fields. Tables 4, 5 and 6 also show that the averaged Nusselt number is only weakly dependent upon $\lambda$. The data presented in these tables show a relatively minor increase in the average Nusselt number at higher values of $\lambda$. This can be explained by noting that $N u_{m}$ is greatly affected by the large local Nusselt numbers around the stagnation point, which are not dependent on the permeability. 
Figure 15 shows the difference between the non-dimensional pressures at the stagnation point and that of other points in the flow. Clearly, the pressure is maximised at the stagnation point and therefore all other points of flow have a relatively lower pressure. For suctiontype transpiration, Fig. 15a shows a monotonic increase in the pressure difference. However, blowing changes this behaviour and results in an initial decrease in the dimensionless pressure for radii close to the surface of the cylinder, followed by a linear increase in the pressure difference. Circumferential distribution of the pressure difference in Fig. 15b reflects the effects of transpiration function and $\lambda$. For the constant transpiration in either of suction or blowing the pressure difference remains constant for the majority of the circumference. Nonetheless, this is not the case for the non-uniform transpiration. Further, as expected, magnifying $\lambda$ leads to the intensification of the pressure difference.

\section{Conclusions}

Fluid flow, temperature field, Nusselt number and the imposed shear stress were investigated theoretically in a stagnation-point flow formed by the impingement of an external flow on a cylinder embedded in porous media. This study extended the existing analyses of external flow impingement on flat porous inserts to a cylindrical configuration. It further introduced different types of transpiration over the surface of the cylinder. This included uniform suction and blowing as well as non-uniform transpiration, which resulted in a non-axisymmetric flow configuration. A semi-similar solution was developed for the flow and temperature fields through reduction of the governing equations to simpler nonlinear differential equations, which were then solved numerically. The major findings of this study can be summarised as follows:

- The radial and axial components of the flow velocity are majorly influenced by the transpiration. Yet, the effects of permeability of the porous medium on the velocity field are less significant.

- External flow Reynolds number appeared to be an important parameter controlling the hydrodynamics of the problem.

- Introduction of non-uniform transpiration resulted in significant variations of flow temperature in the circumferential direction. It was observed that in the case of uniform transpiration, flow temperature around the cylinder remains constant.

- It was shown that increasing Reynolds number reduces the thickness of the thermal boundary layer and hence it significantly modifies the flow temperature distribution in the radial and circumferential directions.

- The averaged Nusselt number was shown to feature a great sensitivity to the transpiration rate. Interestingly, this dependency upon the transpiration is massively intensified at high values of Prandtl number.

The results presented in this work can be further used for validation of the future numerical and theoretical investigations of stagnation-point flows in curved porous media.

Open Access This article is distributed under the terms of the Creative Commons Attribution 4.0 International License (http://creativecommons.org/licenses/by/4.0/), which permits unrestricted use, distribution, and reproduction in any medium, provided you give appropriate credit to the original author(s) and the source, provide a link to the Creative Commons license, and indicate if changes were made. 


\section{References}

Abbassi, A.S., Rahimi, A.B.: Investigation of two-dimensional unsteady stagnation-point flow and heat transfer impinging on an accelerated flat plate. J. Heat Transf. 134(6), 064501 (2012)

Abbassi, A.S., Rahimi, A.B., Hamid, Niazmand H.: Exact solution of three-dimensional unsteady stagnation flow on a heated plate. J. Thermophys. Heat Transf. 25(1), 55-58 (2011)

Alizadeh, R., Rahimi, A.B., Najafi, M.: Magnetohydrodynamic unaxisymmetric stagnation-point flow and heat transfer of a viscous fluid on a stationary cylinder. Alex. Eng. J. 55(1), 37-49 (2016a)

Alizadeh, R., Rahimi, A.B., Najafi, M.: Non-axisymmetric stagnation-point flow and heat transfer of a viscous fluid on a stationary cylinder. Sci. Iran. Trans. B Mech. Eng. 23(5), 2238 (2016b)

Alizadeh, R., Rahimi, A.B., Arjmandzadeh, R., Najafi, M., Alizadeh, A.: Unaxisymmetric stagnation-point flow and heat transfer of a viscous fluid with variable viscosity on a cylinder in constant heat flux. Alex. Eng. J. 55(2), 1271-1283 (2016c)

Bhattacharyya, K., Layek, G.C.: Effects of suction/blowing on steady boundary layer stagnation-point flow and heat transfer towards a shrinking sheet with thermal radiation. Int. J. Heat Mass Transf. 54(1), 302-307 (2011)

Buonomo, B., Lauriat, G., Manca, O., Nardini, S.: Numerical investigation on laminar slot-jet impinging in a confined porous medium in local thermal non-equilibrium. Int. J. Heat Mass Transf. 98, 484-492 (2016)

Cunning, G.M., Davis, A.M.J., Weidman, P.D.: Radial stagnation flow on a rotating cylinder with uniform transpiration. J. Eng. Math. 33(2), 113-128 (1998)

Davey, A.: Boundary-layer flow at a saddle point of attachment. J. Fluid Mech. 10(04), 593-610 (1961)

Feng, S.S., Kuang, J.J., Wen, T., Lu, T.J., Ichimiya, K.: An experimental and numerical study of finned metal foam heat sinks under impinging air jet cooling. Int. J. Heat Mass Transf. 77, 1063-1074 (2014)

Ganesan, P., Palani, G.: Finite difference analysis of unsteady natural convection MHD flow past an inclined plate with variable surface heat and mass flux. Int. J. Heat Mass Transf. 47(19), 4449-4457 (2004)

Hamad, M.A., Pop, I.: Scaling transformations for boundary layer flow near the stagnation-point on a heated permeable stretching surface in a porous medium saturated with a nanofluid and heat generation/absorption effects. Transp. Porous Med. 87(1), 25-39 (2011)

Hamad, M.A.A., Ferdows, M.: Similarity solution of boundary layer stagnation-point flow towards a heated porous stretching sheet saturated with a nanofluid with heat absorption/generation and suction/blowing: a Lie group analysis. Commun. Nonlinear Sci. Numer. Simul. 17(1), 132-140 (2012)

Harris, S.D., Ingham, D.B., Pop, I.: Mixed convection boundary-layer flow near the stagnation point on a vertical surface in a porous medium: Brinkman model with slip. Transp. Porous Med. 77(2), 267-285 (2009)

Hiemenz, K.: Die Grenzschicht an einem in den gleichförmingen Flüssigkeitsstrom eingetauchten geraden Kreiszylinder. Dinglers Polytech. J. 326, 321-410 (1911)

Homann, F.: Der Einfluss grosser Zähigkeit bei der Strömung um den Zylinder und um die Kugel. J. Appl. Math. Mech. Z. Angew. Math. Mech. 16(3), 153-164 (1936)

Howarth, L.: The boundary layer in three-dimensional flow. Part 2. The flow near stagnation point. Lond. Edinb. Dubl. Philos. Mag. 42(7), 1433-1440 (1951)

Ishak, A., Nazar, R., Arifin, N.M., Pop, I.: Dual solutions in mixed convection flow near a stagnation point on a vertical porous plate. Int. J. Therm. Sci. 47(4), 417-422 (2008)

Jeng, T.M., Tzeng, S.C.: Numerical study of confined slot jet impinging on porous metallic foam heat sink. Int. J. Heat Mass Transf. 48(23), 4685-4694 (2005)

Jeng, T.M., Tzeng, S.C.: Experimental study of forced convection in metallic porous block subject to a confined slot jet. Int. J. Therm. Sci. 46(12), 1242-1250 (2007)

Joseph, D.D., Nield, D.A., Papanicolaou, G.: Nonlinear equation governing flow in a saturated porous medium. Water Resour. Res. 18(4), 1049-1052 (1982)

Kokubun, M.A., Fachini, F.F.: An analytical approach for a Hiemenz flow in a porous medium with heat exchange. Int. J. Heat Mass Transf. 54(15), 3613-3621 (2011)

Nazari, M., Shakerinejad, E., Nazari, M., Rees, D.A.S.: Natural convection induced by a heated vertical plate embedded in a porous medium with transpiration: local thermal non-equilibrium similarity solutions. Transp. Porous Med. 98(1), 223-238 (2013)

Nield, D.A., Bejan, A.: Convection in Porous Media, 3rd edn. Springer, New York (2006)

Rahimi, A.B., Saleh, R.: Axisymmetric stagnation-point flow and heat transfer of a viscous fluid on a rotating cylinder with time-dependent angular velocity and uniform transpiration. J. Fluid Eng. 129(1), 106-115 (2007)

Rahimi, A.B., Saleh, R.: Similarity solution of unaxisymmetric heat transfer in stagnation-point flow on a cylinder with simultaneous axial and rotational movements. J. Heat Transf. 130(5), 054502 (2008) 
Rahimi, A.B., Abbassi, A.S.: Three-dimensional stagnation flow and heat transfer on a flat plate with transpiration. J. Thermophys. Heat Tranf. 23(3), 513-521 (2009)

Rohni, A.M., Ahmad, S., Pop, I., Merkin, J.H.: Unsteady mixed convection boundary-layer flow with suction and temperature slip effects near the stagnation point on a vertical permeable surface embedded in a porous medium. Transp. Porous Med. 92(1), 1-14 (2012)

Rohsenow, W.M., Hartnett, J.P., Cho, Y.I.: Handbook of Heat Transfer. McGraw-Hill, New York (1998)

Saleh, R., Rahimi, A.B.: Axisymmetric stagnation-point flow and heat transfer of a viscous fluid on a moving cylinder with time- dependent axial velocity and uniform transpiration. J. Fluid Eng. 126(6), 997-1005 (2004)

Sivasamy, A., Selladurai, V., Kanna, P.R.: Mixed convection on jet impingement cooling of a constant heat flux horizontal porous layer. Int. J. Therm. Sci. 49(7), 1238-1246 (2010)

Thomas, J.W.: Numerical Partial Differential Equations: Finite Difference Methods, vol. 22. Springer, Berlin (2013)

Wang, C.-Y.: Axisymmetric stagnation flow towards a moving plate. AIChE J. 19(5), 1080-1081 (1973)

Wang, C.-Y.: Axisymmetric stagnation flow on a cylinder. Q. Appl. Math. 32(2), 207-213 (1974)

Wong, K.C., Saeid, N.H.: Numerical study of mixed convection on jet impingement cooling in a horizontal porous layer-using Brinkman-extended Darcy model. Int. J. Therm. Sci. 48(1), 96-104 (2009a)

Wong, K.C., Saeid, N.H.: Numerical study of mixed convection on jet impingement cooling in a horizontal porous layer under local thermal non-equilibrium conditions. Int. J. Therm. Sci. 48(5), 860-870 (2009b)

Wu, Q., Weinbaum, S., Andreopoulos, Y.: Stagnation-point flows in a porous medium. Chem. Eng. sci. 60(1), 123-134 (2005) 